Article

\title{
A Data Secured Communication System Design Procedure with a Chaotic Carrier and Synergetic Observer
}

\author{
Alexey Mushenko ${ }^{1}\left(\mathbb{D}\right.$, Julia Dzuba $^{2}$, Alexey Nekrasov ${ }^{1,3,4, *(\mathbb{D}}$ and Colin Fidge ${ }^{5(\mathbb{C}}$ \\ 1 Institute for Computer Technologies and Information Security, Southern Federal University, Chekhova 2, \\ Taganrog 347922, Russia; asmushenko@sfedu.ru \\ 2 JSC Scientific Design Bureau of Computing Systems, 1 Liniya 144-a, Taganrog 347936, Russia; \\ yuliya_dzyuba@mail.ru \\ 3 Department of Radio Engineering Systems, Saint Petersburg Electrotechnical University, Professora \\ Popova 5, Saint Petersburg 197376, Russia \\ 4 Faculty of Electrical Engineering and Informatics, Technical University of Košice, Letná 9, \\ 04200 Košice, Slovakia \\ 5 Science and Engineering Faculty, Queensland University of Technology (QUT), Gardens Point Campus, \\ Brisbane, QLD 4001, Australia; c.fidge@qut.edu.au \\ * Correspondence: alexei-nekrassov@mail.ru; Tel.: +7-8634-360-484
}

Received: 7 February 2020; Accepted: 15 March 2020; Published: 18 March 2020

check for updates

\begin{abstract}
We have considered the problem of secure communication by means of chaotic carrier communication channels. Data protection is provided if the signal in the communication channel looks like a stochastic or noisy one, i.e., by the steganography property, and also by using advanced and secured approaches of mixing a useful signal into a chaotic carrier. The problem is to find effective and efficient techniques for useful signal reconstruction at the receiver side. We firstly use a synergetic nonlinear mathematical observer to recover the two useful signals transmitted simultaneously over a single communication channel. Compared to a known observer, the synergetic one operates with initial nonlinear models, i.e., it may be applied directly to chaotic systems. In that system structure, we consider the useful signals as unobservable variables and estimate them by the given model of a chaotic generator. A chaotic data transmission system with two channels is demonstrated; the presented mathematical procedure explains the steps of synergetic two-channel observer design. Computer simulation results prove the viability of our approach. The proposed data transmission scheme and the observer design procedure provide effective transmission and reconstruction of informational signals. Reconstruction error is up to approximately $25 \%$ but has a very short pulses shape and could be eliminated with further analog or digital filtering algorithms. As an example application, simulation of image transmission and recovery is demonstrated. The study's results may be used as a basis for further research into secure data transmission system design.
\end{abstract}

Keywords: synergetic control theory; observer; dynamic chaos; secured communication; chaotic carrier; chaotic parameter nonlinear modulation

\section{Introduction}

Dynamic chaos as a phenomenon has attracted researchers' attention since the well-known works of Lorenz [1,2]. Chaotic systems have found application in the study of chemical reactions, phenomena in medicine, biology, economics, sociology, engineering, etc. [2-8]. Chaotic systems and the phenomena of dynamic chaos are also used in the development of communication systems [9-12]. Development of new methods of data protection is always a state-of-the-art problem. Traditional approaches are 
digital and/or analog signals hardware and software encryption with various algorithms. Another advanced approach is use of chaotic oscillation generators instead of a van der Pol oscillator as a carrier oscillation generator in data transmission systems. In such a case the unpredictable nature of a chaotic carrier to an external listener becomes a protective tool itself. It should be noted that the chaotic carrier dramatically complicates a communication system, producing a lot of engineering problems. The most important problems are: (i) introduction of the informational signal into the chaotic carrier at the transmitter side while providing a sufficient security level; and (ii) successful recovery/reconstruction of the information signal at the receiver side. Secondary problems may include transmitter and receiver systems synchronization, signal reconstruction accuracy, etc.

There are various methods for protecting a useful signal with chaotic oscillations [12]. These methods differ both in the way of introducing a useful signal into a chaotic one in a communication channel and in the way of recovering a useful signal on the receiver side. The most promising methods are nonlinear mixing (or chaotic generator parameter nonlinear modulation) of a useful signal into the structure of a chaotic generator and adaptive methods of signal reception [12,13].

In our study, we combine these advanced approaches and explore a chaotic carrier two-channel data transmission system using nonlinear parametric modulation at the transmitter side and a two-channel observer for useful signal estimation at the receiver side.

The main contributions of this study are summarized as follows:

- Here we firstly present a design procedure for a two-channel nonlinear synergetic observer that we used as an adaptive method of signal reception in chaotic carrier communication system;

- The presented design procedure is implemented for Rössler generator;

- We built a chaotic communication system viable structure based on the combination of chaotic generator nonlinear parametric modulation, method of global reconstruction of a dynamic system and nonlinear synergetic observer;

- Application of synergetic observer chaotic carrier communication system for image transmission.

The article is organized as follows. Section 2 presents an overview of various methods of secured signal transmission using the chaotic generator as a signal carrier including ones that use parametric modulation and various techniques of signal reconstruction on the receiver side including observer design methods. Section 3 presents the methodology of our approach considered here (Section 3.1), security analysis of the encoded transmission system (Section 3.3), a system design procedure algorithm with a design example (Sections 3.4 and 3.5) as well as an overall system computer simulation (Section 3.6). To compare the obtained results, the chaotic system was estimated using the least squares method in Section 3.7. In Section 4.1, some hardware system implementation options on an Arduino, Raspberry Pi and FPGAs are discussed based on examples from other studies described earlier [14-20]. Besides Section 4 provides examples of system software implementation, including an example of digital data software transmission (Section 4.2) and secured image transmission via the designed system in Section 4.3. Discussion and conclusions as well as the study's future directions are presented in Sections 5 and 6.

\section{Related Works and Methods Overview}

The overall issues and approaches of chaotic communications when using chaotic modulation schemes were briefly described by Riaz and Ali [21]. According to their study [21], the most common modulation schemes are: chaos shift keying (CSK); differential chaos shift keying (DCSK); additive chaos modulation (ACM); and multiplicative chaos modulation (MCM). CSK and DCKS provide secure communication by shifting between two identical or different chaotic generators. ACM and MCM provide mixing of the useful signal directly into the mathematical model of a chaotic generator in a linear (additive) or nonlinear (multiplicative) manner. In all of these schemes, one of the data secure factors is a chaotic carrier signal frequency ten times greater than the useful signal frequency or more. ACM with a combination of MCM was explored by Kevin Short in the 1990s [22] in several 
papers, where he proves that this kind of combined modulation does not provide security for a 3rd order system. In addition, we note that using only MCM is a more secure approach due to nonlinear mixing of the useful signal and MCM as a secure scheme is thus widely used in much research as indicated below. Different approaches and applications of chaotic data communication systems design have been illustrated in several recent papers [23-26]. Examples of CSK and DCSK were presented by Yang et al. [23] and Cheng et al. [24]. Chaotic generator parameter modulation was used by Wang [25] as well as an adaptive synchronization approach based on Lyapunov stability theory for the receiver design procedure. The problem of such approaches is how to select the Lyapunov function in the general case of chaotic communication system design. An example of chaotic phase modulation of couple modified Rössler oscillators for transmission of analog information was presented by Isaeva et al. [26]. Thus MCM could be the most simple and secure configuration of the transmitter block of a chaotic communication system but it requires a powerful procedure to reconstruct a useful signal at the receiver side.

The use of a state observer for reconstructing a useful signal in information transmission systems has been shown previously by Andrievsky and Fradkov [27,28], Pogromsky [29], and Tyukin et al. [30]. Moreover, the observer-based approach is widely used in recent works of various authors in chaos communication system design and other applications. For example, Chien and Chen [31] demonstrate a method of estimating the unmeasured state variable to derive a plant dynamics full-order model, but only for a linear system. Wei and Guo [32] built a disturbance observer to eliminate noise in an industrial system. A geometric approach to transform the original strongly detectability system into a strongly observable and detectable subsystem was used by Zhang and Zhu [33]. The problem of observer-based synchronization was explored by Xiao and Pan [34]. Another most promising approach of secure data transmission systems control and synchronization is observer design for sliding mode control (SMC) as explored by Datcu et al. [35]. Finally, Moysis et al. [36] explored the problem of line equilibrium chaotic system synchronization based on state observer design for a rectangular system.

However, the state observers used in earlier research [27-37] reduce to the synergetic state observers [38-40] used in our work. Table 1 summarizes distinctive features of some of the explored reconstruction techniques to provide a more visible comparison between different works and approaches in the field and highlight the merit of a synergetic state observer.

Table 1. Signal reconstruction techniques features comparison.

\begin{tabular}{cccccc}
\hline Reconstruction technique & $\begin{array}{c}\text { Two Channel } \\
\text { Estimation }\end{array}$ & $\begin{array}{c}\text { Lyapunov } \\
\text { Function } \\
\text { Definition }\end{array}$ & $\begin{array}{c}\text { Application to } \\
\text { Nonlinear } \\
\text { Objects }\end{array}$ & $\begin{array}{c}\text { Observer } \\
\text { structure }\end{array}$ & $\begin{array}{c}\text { Need to Find a } \\
\text { Control Law }\end{array}$ \\
\hline Luenberger observer [32] & No & Not needed & Limited & Rigid/Postulated & Yes \\
Kalman filter [31] & No & Not needed & Limited & Rigid/Postulated & Yes \\
Chaotic synchronization [25,27] & No & Required & Yes & Rigid/Postulated & Yes \\
Adaptive observer [27-29] & No & Not needed & Yes & Rigid/Postulated & No \\
SMC observer [35] & No & Not needed & Yes & Rigid/Postulated & Yes \\
Reduced order observer [36,37] & No & Required & Yes & Rigid/Postulated & No \\
Synergetic observer [39] & Yes & Not needed & Yes & Object-defined & No \\
\hline
\end{tabular}

So, as described in Table 1 and beyond, the key advantages and main distinctive features in the design procedure of a synergetic observer in comparison with other observers are:

- the structure of classical observers is rigid, i.e., the relationship between its variables are initially postulated but not formed during the design process for a particular object;

- the synergetic observer's structure is formed directly during the design procedure, so the observer has to be specially designed for particular object model, but the design procedure is more complicated and should be defined in every case;

- the synergetic observer's designed structure includes the right-hand sides of the equations of the chaotic generator, so it allows taking into account all the properties of a chaotic generator and the relationship between its variables. 


\section{Methodology and the System Design Procedure}

\subsection{General Methodology}

We explored a nonlinear chaotic oscillations generator as a source of the carrier signal in a data secured communication system. Our goal was to transmit a useful signal over this chaotic carrier. To modulate our useful signal, we introduced it as a function directly into the structure of the generator of chaotic oscillations, so the output would be a highly-secured analog signal. Then this encoded signal entered the communication channel. To restore our useful signal on the receiver side we applied a known approach of synergetic nonlinear state observer design. To do so we treated our useful signal as an unknown variable and applied the designed observer for its estimation and reconstruction.

The adaptive reception methods used in this work, including the work of mixing a useful signal directly into the structure of the chaotic generator model, have been considered in the works of Professor V.S. Anishchenko [13] and studied in several earlier papers [27-30].

Within the framework of these previous methods, in our work two of the constant coefficients of the chaotic generator model were replaced by functions whose arguments were the sum of the initial coefficient and the useful signal that varies over time. Thus, the useful signals were embedded directly into the structure of the chaotic generator itself and the resulting chaotic oscillations carried hidden information within these useful signals. This approach to mixing useful signals, not into the resulting chaotic oscillations, but directly into the generator itself in a nonlinear, multiplicative manner only, increased the stealth and security of the system. The innovation of this work was to restore the useful signals on the receiver side if there was information about the model of the chaotic carrier signal generator and its parameters, the modulated useful signal parameter, which non-linear mixing was performed, and what was considered an unobservable variable, that was subject to recovery by a synthesized state observer. To restore useful signals, we used a synergetic nonlinear observer of state variables, proposed in the works of the scientific school of Professor A.A. Kolesnikov [38-40], to which the authors of this study belong; such an observer is able to work with a specific generator of deterministic chaos. The procedure for using a synergetic observer to reconstruct a signal with a chaotic carrier was presented earlier [39]. Our synergetic observer was based on the method of analytical design of an aggregated regulator (ADAR) that was first presented by Kolesnikov [41] and later extended to many applications in several papers and studies (e.g., [38,40]) within a framework of synergetic control theory. In previous research [42,43], a synergetic observer application for a single-channel system with a chaotic carrier was shown. In this paper, a new design procedure is presented that works with a system employing two-channel transmission of useful signals.

So, the communication system draft structure may be presented as in Figure 1. In the following subsections, we will specify each block of Figure 1 to build a communication system reflecting above mentioned general methodology.

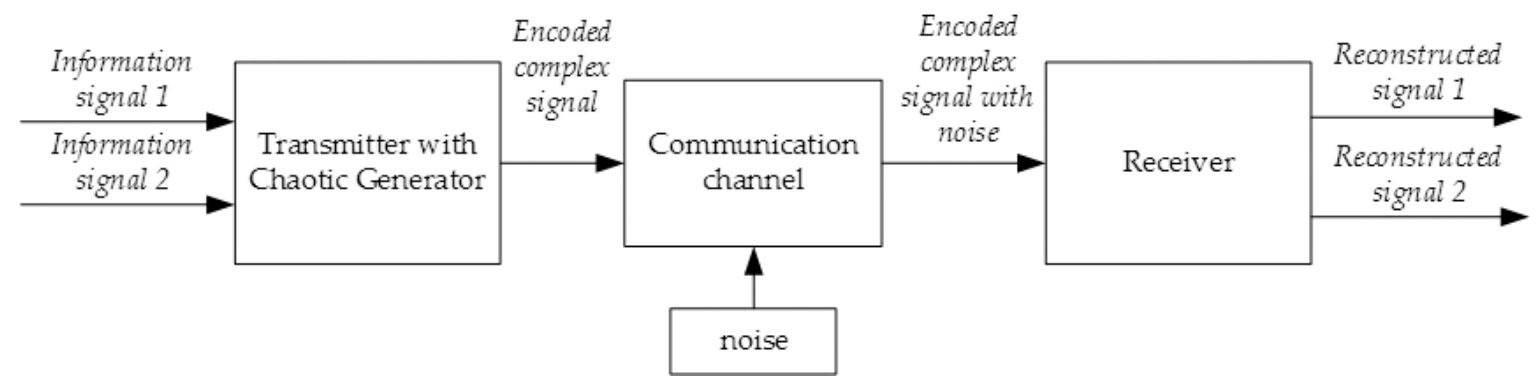

Figure 1. General block diagram of a chaotic communication system.

As an approach to building communication systems with a chaotic carrier, chaotic synchronization is used in many studies. On the contrary, our system will allow not using chaotic synchronization and will provide a simpler circuit on the receiver's side. 


\subsection{Chaotic Generator Mathematical Model and Two-Channel Chaotic Transmitter Block Diagramm}

Let us consider a dynamic chaos generator based on a "strange" attractor of Rössler [44], which describes the dynamic regimes by the following nonlinear differential equations

$$
\begin{aligned}
& \dot{x}(t)=-y-z ; \\
& \dot{y}(t)=x+a y ; \\
& \dot{z}(t)=b+x z-c z,
\end{aligned}
$$

where $x, y$ and $z$ are state variables, and $\mu^{0}=[a, b, c]$ is the vector of constant parameters.

In accordance with the method of global reconstruction of dynamical systems [13], by using the following change of variables

$$
\begin{aligned}
& x=X-a Y \\
& y=Y \\
& z=a X-Y-Z
\end{aligned}
$$

we transform Equation (1) to the following form

$$
\begin{aligned}
& \dot{X}(t)=Y ; \\
& \dot{Y}(t)=Z ; \\
& \dot{Z}(t)=f\left(X, Y, Z, \mu^{0}\right)
\end{aligned}
$$

where

$$
f\left(X, Y, Z, \mu^{0}\right)=(a-1) X-Y+(a-1) Z-a X^{2}-a Y^{2}+\left(a^{2}+1\right) X Y+c(a X-Y-Z) .
$$

The Rössler generator mathematical model in the form of Equations (3) and (4) provides transmission of variables $x(t), y(t)$ and $z(t)$ over a single communication channel with only one new variable $Z(t)$. In verifying that Equations (1) and (3) are equivalent, in Figure 2 we show attractors of initial Rössler model (1) and reconstructed model (3).

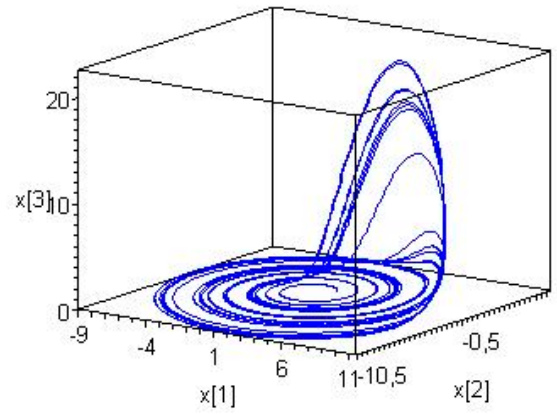

(a)

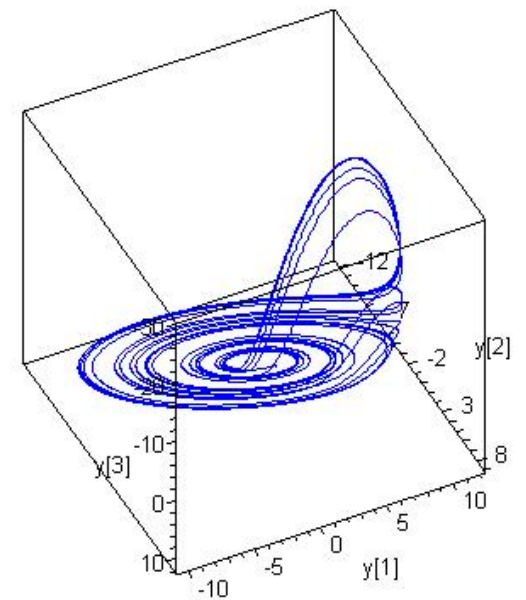

(b)

Figure 2. Rössler chaotic generator attractor for (a) initial and (b) reconstructed systems.

Thus, the transformed system (3) in the general case contains three unknown parameters $a, b$, and $c$, which can be found by constructing a synergetic observer. 
As we decided to use a two-channel useful data transmission system, we perform modulation of parameters $a$ and $c$ from drive signals so that their value remains within the chaotic dynamic system model. This gives us the new control parameters of the Rössler system as follows.

$$
\begin{aligned}
& a^{*}(t)=a+\mu_{1}(t) \\
& c^{*}(t)=c+\mu_{2}(t) .
\end{aligned}
$$

The condition of chaotic mode existence in the system should be determined in the following intervals.

$$
\left\{\begin{array}{c}
0.15 \leq a \leq 0.25 \\
b=0.2 \\
5 \leq c \leq 6
\end{array}\right.
$$

So, the transmitter MATLAB/Simulink model structure according to model (3) had the form as in Figure 3. In Figure 3 the block $d z / d t$ consists function (4), and inputs $a(t)$ and $c(t)$ were modulated with useful signals according to (5).

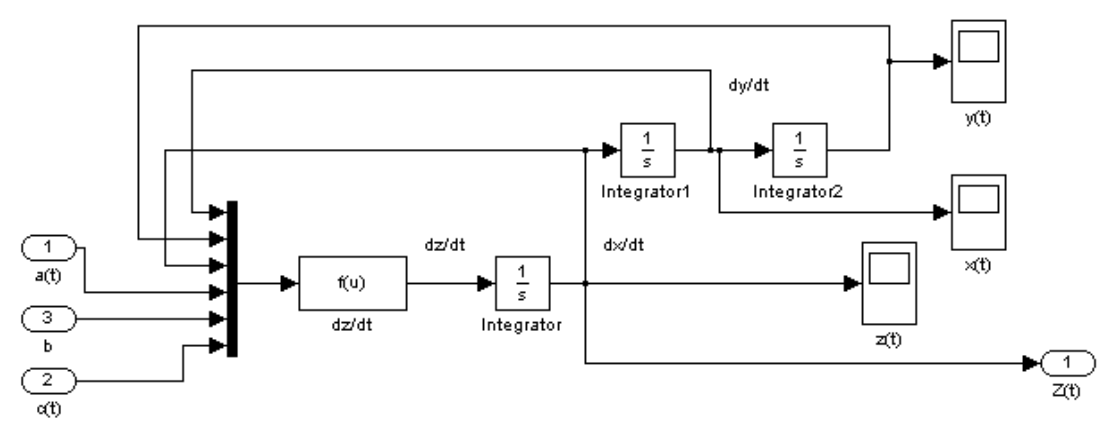

Figure 3. MATLAB/Simulink model of the transmitter block.

Figure $4 \mathrm{a}, \mathrm{b}$ display initial useful signals $a(t)$ and $c(t)$ with rectangular pulses at the inputs of the transmitter. Figures 5 and 6 display the nature of the encoded generator state variables. Depending on the transmission system structure the different numbers of physical transmission lines may be enabled. In our case we used only single line transmission, so state variables $X(t)$ and $Y(t)$ were included in state variable $Z(t)$ and only $Z(t)$ signal (presented in Figure 6) is transmitted via the communication channel. Then the signal in the communication channel was under the influence of external disturbances.

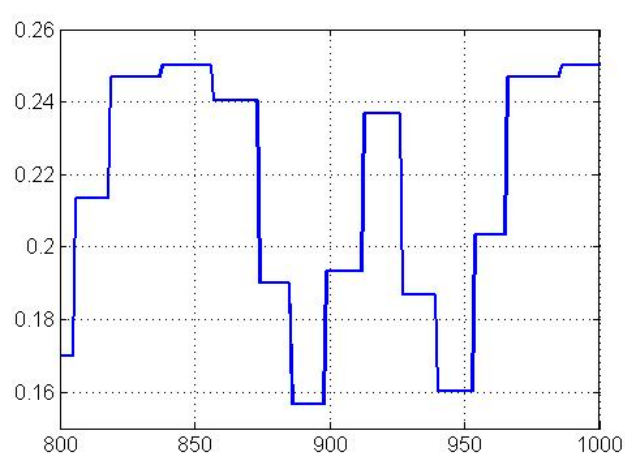

(a)

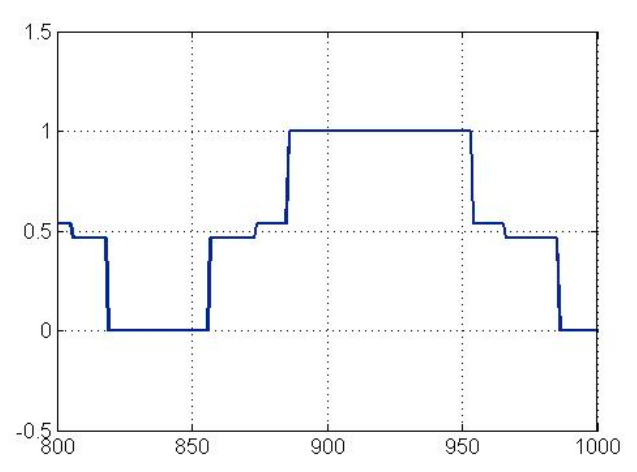

(b)

Figure 4. Initial useful signals with rectangular pulses at the inputs of transmitter: (a) useful signal $a(t)$; (b) useful signal $c(t)$. 


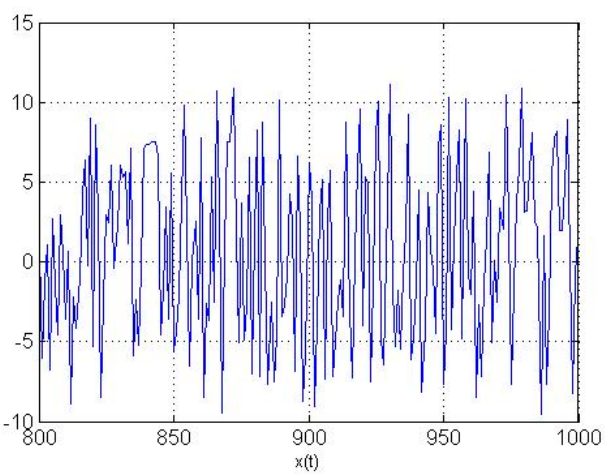

(a)

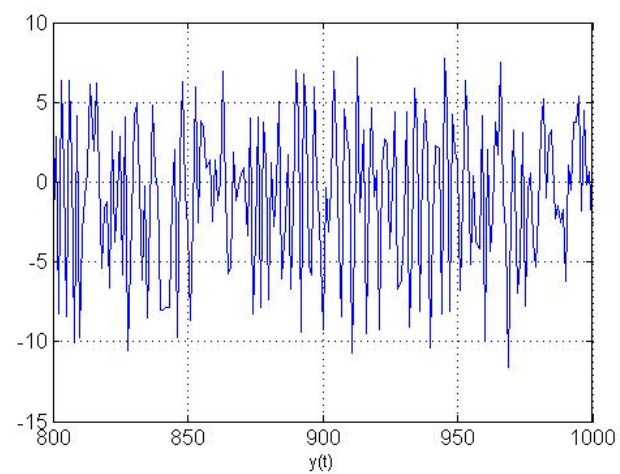

(b)

Figure 5. Encoded state variables at the transmitter: (a) state variable $X(t)$; (b) state variable $Y(t)$.

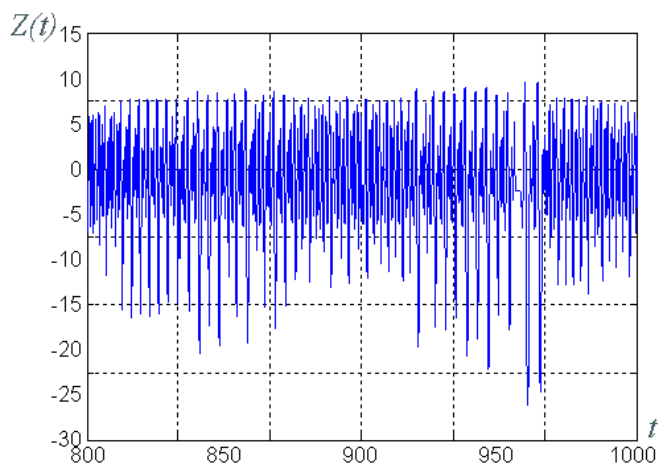

Figure 6. Encoded signal with rectangular pulses in the communication channel (state variable $Z(t))$.

\subsection{Security Analysis of Chaotic Carrier Data Transmission System with Parameters Nonlinear Modulation}

In the previous sections, we described different schemes for mixing a useful signal with a chaotic one. In particular, the works of Short [22] indicated in Section 2 show the vulnerability of systems with additive parametric modulation. In his works, it was shown that it is possible to create a filter that selects a useful (i.e., deterministic) signal from a chaotic carrier. In this regard, the security analysis of systems in Section 3.2 with multiplicative parametric modulation is valid. It is obvious that nonlinear mixing and the system (1) global reconstruction into Equations (3) and (4) will provide a more complex waveform for $Z(t)$, but it is necessary to prove this mathematically. For this purpose, we applied the signal $Z(t)$ to the communication channel with a multiplicatively mixed some useful periodic signal (hereinafter, a useful signal transmission) and without a mixed useful signal (hereinafter, an idle transmission). To analyze these transmissions, we used the standard signal processing functions from the MATLAB: autocorrelation and Fourier transform.

\subsubsection{Autocorrelation Analysis}

Autocorrelation is the correlation of a signal with itself, but with some time delay [45]. This estimation is also called a sequential correlation. The MATLAB system has a special function for obtaining correlograms, namely the xcorr function.

Figure 7a,b shows that the shape of the correlograms did not indicate the presence of a useful signal in a chaotic carrier. Moreover, the shape of the correlogram of a completely random signal (Figure 8) also did not differ from the correlograms of deterministic chaos. Thus, analysis using correlograms confirmed the possibility of using this method to protect data transmission. Moreover, this ensured the steganography of the system, i.e., concealment from an external listener of the very fact that we were transmitting a useful signal. 


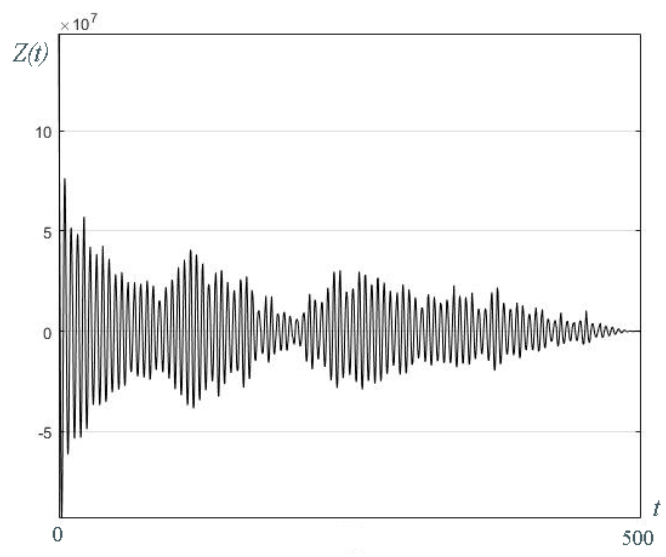

(a)

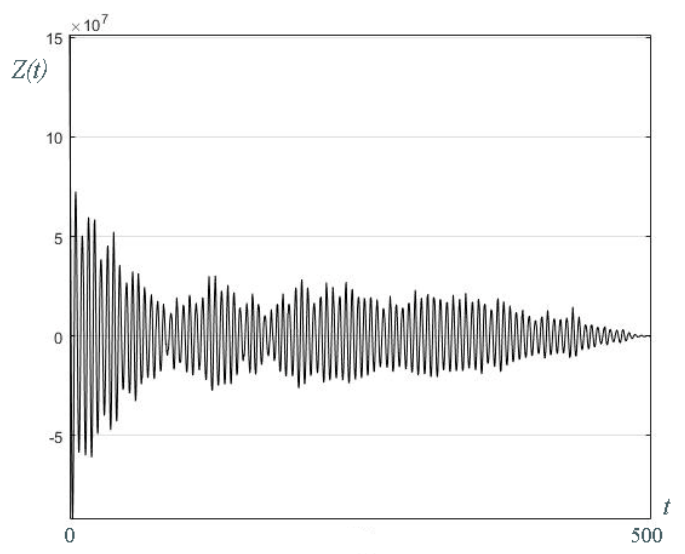

(b)

Figure 7. Correlograms of $Z(t)$ : (a) idle transmission (without useful signal); and (b) transmission with useful signal (nonlinear, multiplicative modulation).

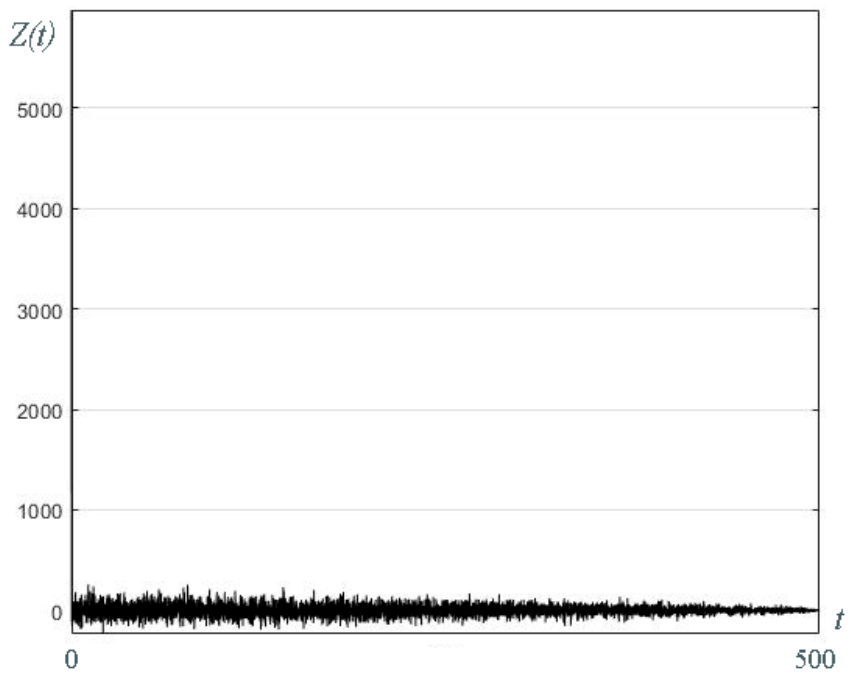

Figure 8. Correlogram of a random signal.

\subsubsection{Fourier Series Analysis}

Fourier series analysis allowed us to demonstrate the non-obvious frequency of the signal. This is so because the Fourier series result is the signal frequency spectrum. For extra analysis, we used Savitzky-Golay filtering. The red lines in Figures 9 and 10 show the filtered signal.

Here, according to Figure $9 \mathrm{a}, \mathrm{b}$, it is also impossible to find significant differences between the results for transmission with a useful signal and without a useful signal. However, the analysis of the non-deterministic random signal (Figure 10) revealed a significant difference in the shape of the signal from the deterministic chaotic one (Figure 9). The latter makes it possible to identify the fact of transmitting a useful signal or the fact of the presence of a useful signal generator in the data transmission medium (even in idle mode transmission). Thus a steganography property, in this case, could not be provided. However, in real systems, there always exist some deterministic signals (interference from other communication channels, etc.). This means it is virtually impossible to obtain an ideal non-deterministic noise in the communication channel in the absence of transmission of a useful signal. 


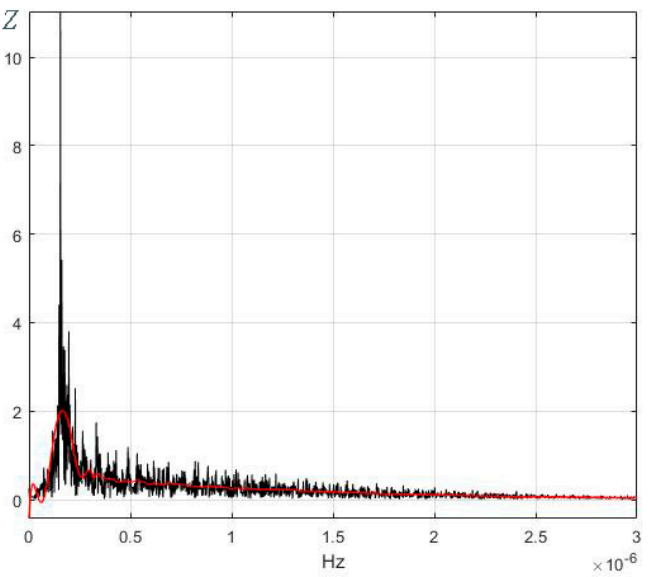

(a)

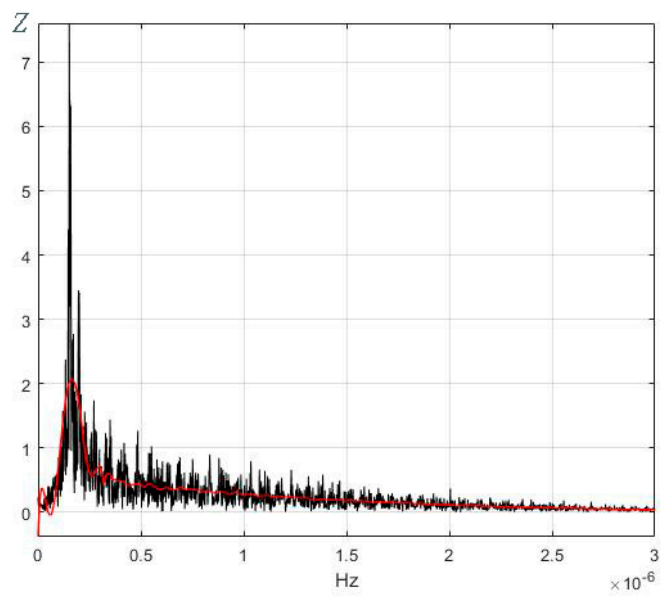

(b)

Figure 9. Fourier series plots for $Z(t)$. Black line: Fourier series; red lines: Savitzky-Golay filter; (a) idle transmission; and (b) transmission with useful signal.

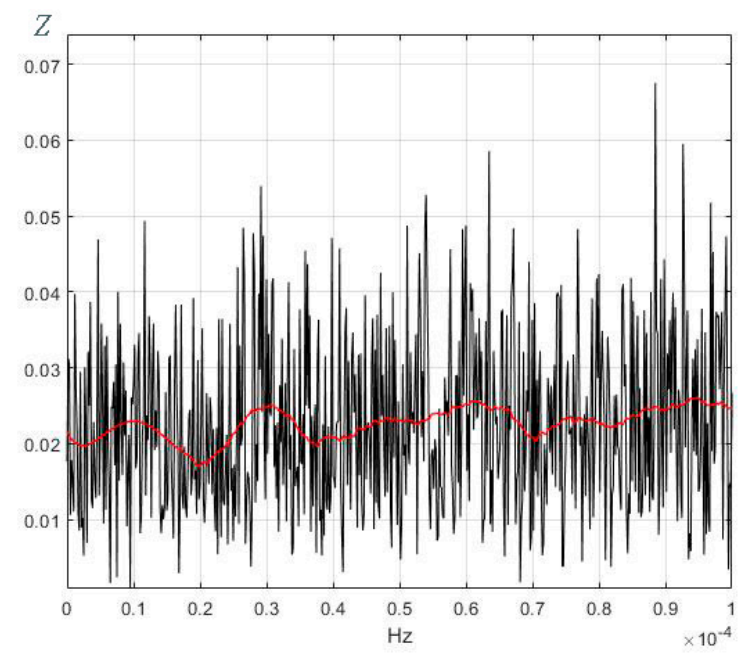

Figure 10. Fourier series plot for random signal (white noise). Black line: Fourier series; red lines: Savitzky-Golay filter.

Thus, the above analysis showed that the multiplicative modulation of a chaotic carrier does not allow us to separate the useful signal from the carrier and does not even allow us to establish the fact of transmission for an external listener. The above analysis using autocorrelation and Fourier series is the simplest analysis. The problem requires other solutions, however, the result above allows us to conclude that the used approach is viable for data protection.

In the following sections, we considered the procedure for reconstructing the useful signal on the receiver side using the methods and approaches of synergetic control theory [38-41].

\subsection{Overall Steps of Synergetic Observer Design Procedure}

The synergetic observer design consists in using of some mathematical algorithm that connects the values of the observed (measured) variables with the estimates of the unobserved variables, i.e., the observer performs restoring the complete state variables vector from the available signal and some a priori information. In the design procedure of a synergetic observer, both disturbing and setting actions, as well as object parameters, can act as unobserved variables. 
In the general case, the mathematical model of an extended system consists of observable and unobservable terms, and is represented as [40]:

$$
\begin{aligned}
& \dot{x}(t)=g_{0}(x)+G_{1}(x) y ; \\
& \dot{y}(t)=h_{0}(x)+H_{1}(x) y,
\end{aligned}
$$

where $x$ is the observed state variables vector, $\operatorname{dim} x=n ; y$ is the vector of unobservable variables (unmeasured physical variables, parameters, setting and disturbing actions, etc.), $\operatorname{dim} y=k ; g_{0}$ and $\boldsymbol{h}_{0}$ are the continuous nonlinear vector functions, $\operatorname{dim} g_{0}=(n \times 1), \operatorname{dim} \boldsymbol{h}_{0}=(k \times 1), \boldsymbol{G}_{1}(\boldsymbol{x})$ is the functional matrix, $\operatorname{dim} \boldsymbol{G}_{1}=(n \times k), \boldsymbol{H}_{1}(\boldsymbol{x})$ is the functional matrix, $\operatorname{dim} \boldsymbol{H}_{1}=(k \times k)$.

The main steps of the synergetic observer design procedure [40] are:

Main Step 1. Synthesis of a synergetic observer. The synthesis task is to construct an asymptotic estimation $\hat{y}$ of the vector $y$ from the observed values of the vector $x$.

Main Step 2. Replacing unobserved variables $y$ with their asymptotic estimates $\hat{y}$.

In our case the equations of the asymptotic observer have the form:

$$
\begin{aligned}
& \dot{z}=\mathbf{L z}-\boldsymbol{L} \int_{0}^{\boldsymbol{x}} \Gamma(\boldsymbol{x}) d x-\boldsymbol{h}_{0}(\boldsymbol{x})+\Gamma(\boldsymbol{x}) \boldsymbol{g}_{0}(\boldsymbol{x}) \\
& \hat{\mathbf{y}}=\int_{0}^{x} \Gamma(\boldsymbol{x}) d x-\boldsymbol{z},
\end{aligned}
$$

where $z$ is the observer state variable vector; $L$ is the numerical matrix, $\operatorname{dim} L=(k \times k)$, selected based on the observer's stability conditions; $\Gamma(\boldsymbol{x})$ is the functional matrix, $\operatorname{dim} \Gamma=(k \times n), \Gamma(\boldsymbol{x})$ is the determined from the following matrix equation:

$$
H_{1}(x)-L=\Gamma(x) G_{1}(x) .
$$

\subsection{Two-Channel Synergetic Observer Analytical Design Procedure and Two-Channel Receiver Block Diagramm}

Here we consider the design procedure for a synergetic state observer for the parameters of the Rössler chaotic generator [44]. The Rössler system is described by a system of nonlinear differential Equation (1).

As control parameters adopted by the parameters $a$ and $c$, let us design the synergetic observer by setting parameter $b$ as "known" [13,26-29]. According to overall design procedure [40], we replace the unknown parameters with their dynamic models

$$
\begin{aligned}
& \dot{w}_{1}(t)=0, \\
& \dot{w}_{2}(t)=0,
\end{aligned}
$$

the solutions of which are $w_{1}(t)=$ const and $w_{2}(t)=$ const, and reflect the quick change in time of parameters $a$ and $c$.

Then, we extend the system of Equations (1) to

$$
\begin{aligned}
& \dot{x}(t)=-y-z ; \\
& \dot{y}(t)=x+a y ; \\
& \dot{z}(t)=b+x z-c z ; \\
& \dot{w}_{1}=0 ; \\
& \dot{w}_{2}=0,
\end{aligned}
$$

where $w_{1}$. is the dynamic state variable model of parameter $a$, and $w_{2}$ is the dynamic state variable model of parameter $c$.

Thus, in System (9) the observable variables are $x, y$ and $z$, and the unobservable variables are $w_{1}$ and $w_{2}$. 
We introduce variables $\hat{w}_{1}$ and $\hat{w}_{2}$, which are estimates of the parameters $w_{1}$ and $w_{2}$ respectively. To find them we define the vector of the macro variable $\Psi(t)$ by the expression

$$
\Psi=\left[\begin{array}{l}
w_{1}-\hat{w}_{1} \\
w_{2}-\hat{w}_{2}
\end{array}\right]
$$

Then, we write down the reduction equations

$$
\begin{aligned}
& \hat{w}_{1}=Q_{1}(x, y, z)+v_{1}, \\
& \hat{w}_{2}=Q_{2}(x, y, z)+v_{2},
\end{aligned}
$$

where $Q_{1}(x, y, z)$ and $Q_{2}(x, y, z)$ are the unknown functions of the observed system state variables in Equations (9), and $v_{1}$ and $v_{2}$ denote the state of the dynamic observer.

Differentiating Equation (11) with respect to time produces

$$
\begin{aligned}
& \frac{d \hat{w}_{1}}{d t}=\frac{\partial Q_{1}(x, y, z)}{\partial x} \frac{d x}{d t}+\frac{\partial Q_{1}(x, y, z)}{\partial y} \frac{d y}{d t}+\frac{\partial Q_{1}(x, y, z)}{\partial z} \frac{d z}{d t}+\frac{d v_{1}}{d t} \\
& \frac{d \hat{w}_{2}}{d t}=\frac{\partial Q_{2}(x, y, z)}{\partial x} \frac{d x}{d t}+\frac{\partial Q_{2}(x, y, z)}{\partial y} \frac{d y}{d t}+\frac{\partial Q_{2}(x, y, z)}{\partial z} \frac{d z}{d t}+\frac{d v_{2}}{d t} .
\end{aligned}
$$

The macro variable in Equation (10) must satisfy the following functional equation

$$
\dot{\Psi}(t)+L \Psi=0
$$

where $L=\left[\begin{array}{ll}L_{11} & L_{12} \\ L_{21} & L_{22}\end{array}\right]$ is the coefficient matrix, which provides the stability of Equations (13) and defines the observer's dynamics.

Then, from Equation (13) with regard to Equations (9) to (12), we obtain

$$
\begin{aligned}
& -\frac{\partial Q_{1}(x, y, z)}{\partial x}(-y-z)-\frac{\partial Q_{1}(x, y, z)}{\partial y}\left(x+w_{1} y\right)-\frac{\partial Q_{1}(x, y, z)}{\partial z}\left(b+x z-w_{2} z\right)- \\
& -\frac{d v_{1}}{d t}+L_{11}\left(w_{1}-\hat{w}_{1}\right)+L_{12}\left(w_{2}-\hat{w}_{2}\right)=0, \\
& -\frac{\partial Q_{2}(x, y, z)}{\partial x}(-y-z)-\frac{\partial Q_{2}(x, y, z)}{\partial y}\left(x+w_{1} y\right)-\frac{\partial Q_{2}(x, y, z)}{\partial z}\left(b+x z-w_{2} z\right)- \\
& -\frac{d v_{2}}{d t}+L_{21}\left(w_{1}-\hat{w}_{1}\right)+L_{22}\left(w_{2}-\hat{w}_{2}\right)=0 .
\end{aligned}
$$

Since the equations of the observer cannot contain the unobservable state variables, we have the following expressions

$$
\left\{\begin{array}{c}
-\frac{\partial Q_{1}(x, y, z)}{\partial y} y+L_{11}=0, \\
\frac{\partial Q_{1}(x, y, z)}{\partial z} z+L_{12}=0, \\
-\frac{\partial Q_{2}(x, y, z)}{\partial y} y+L_{21}=0, \\
\frac{\partial Q_{2}(x, y, z)}{\partial z} z+L_{22}=0 .
\end{array}\right.
$$

Since the system of Equation (15) is of the fourth order, but has six unknown terms, starting from the stability conditions of the observer, and taking into account Equation (9), we choose

$$
L_{11}=\alpha y^{2}, L_{12}=0, L_{21}=0, L_{22}=\alpha z^{2} \text {. }
$$

where $\alpha$ is the observer tuning parameter introduced to determine the rate of estimation. Then

$$
\begin{aligned}
& \frac{\partial Q_{1}}{\partial y}=\frac{L_{11}}{y}=a y, \frac{\partial Q_{1}}{\partial z}=-\frac{L_{12}}{z}=0, \\
& \frac{\partial Q_{2}}{\partial y}=\frac{L_{21}}{y}=a y, \frac{\partial Q_{2}}{\partial z}=-\frac{L_{22}}{z}=-a z,
\end{aligned}
$$


and

$$
\begin{aligned}
& Q_{1}(x, y, z)=\frac{1}{2} \alpha y^{2}, \\
& Q_{2}(x, y, z)=-\frac{1}{2} \alpha z^{2} .
\end{aligned}
$$

Thus, taking into account Equations (17) and (18), the observer's Equation (14) take the following form:

$$
\begin{aligned}
& \dot{v}_{1}=-\alpha x y-\alpha y^{2} \hat{w}_{1}, \\
& \dot{v}_{2}=\alpha z(b+x z)-\alpha z^{2} \hat{w}_{2},
\end{aligned}
$$

where the estimates of variables $\hat{w}_{1}$ and $\hat{w}_{2}$ are calculated according to Equation (11).

So, the receiver MATLAB/Simulink model structure according to model (5), (19) has the form shown in Figure 11.

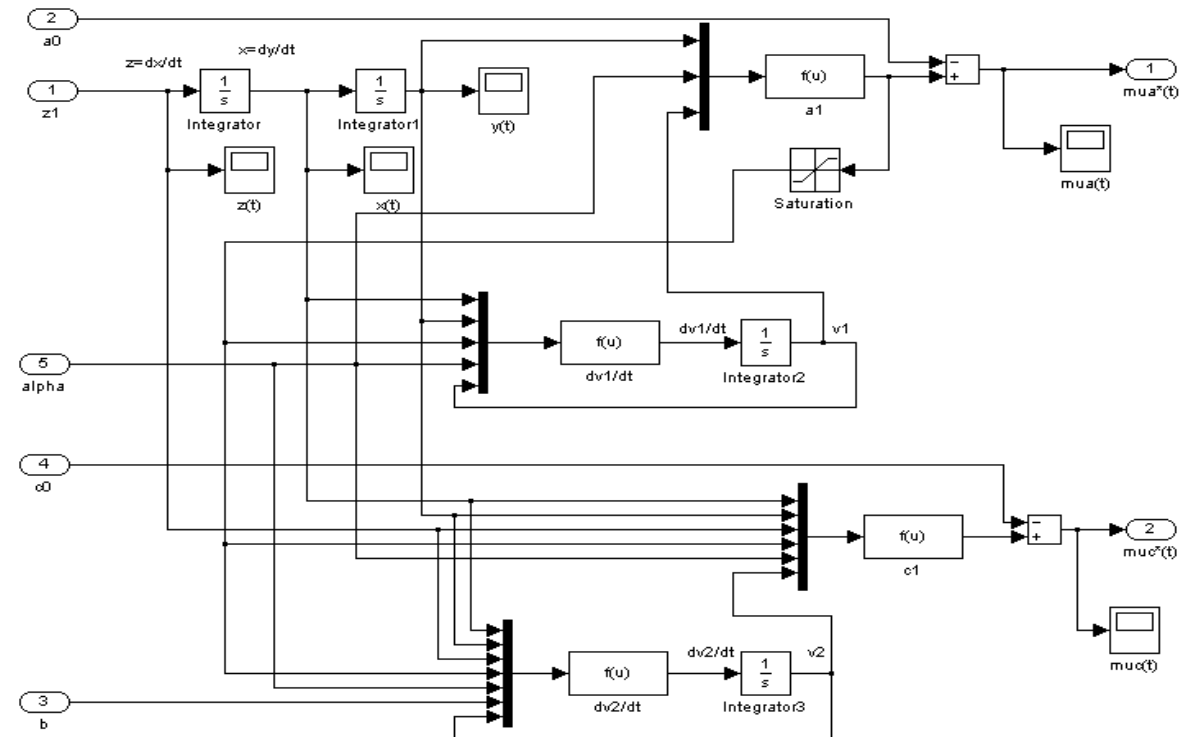

Figure 11. MATLAB/Simulink model of the receiver (two-channel observer).

Figure 11 displays our MATLAB/Simulink model of the receiver with the two-channel synergetic observer and implements the initial model and observer equations from Section 3.5. In Figure 11 the blocks $a 1$ and $c 1$ consist function (18).

\subsection{Building of the Data Trasmission System Model and Overall System Computer Simulation}

In MATLAB/Simulink, we have built a model (Figure 12) reflecting a two-channel chaotic data transmission system (Figures 3 and 11) and provided a source and reconstructed signals as in Figure 13. Figure 6 presents the encoded signal into the communication channel.

The following parameters were used in Figure 12 model simulation:

- Rössler system parameter $b=0.2$; and

- the parameter of the synergetic observer, which determines the rate of estimation $\alpha=-0.1$.

Figures 3, 11 and 12 describe our virtual test bed in MATLAB/Simulink.

Figure 12 displays the overall secure communication system model with a two-channel synergetic observer without presenting the transmitter and receivers in detail. In the figure, the useful signals were generated in the "Information signal" block 1 . Then the useful signals were directed to the "Transmitter" block 2 where they were mixed into the chaotic generator mathematical model followed with mapping into unified variable $Z(t)$. Then $Z(t)$ entered the "Communication channel" block 3 , where $Z(t)$ was admixed with disturbances emulated with the MATLAB/Simulink block "Uniform Random number". Block 5 in Figure 12 was the receiver side with a synergetic observer. 


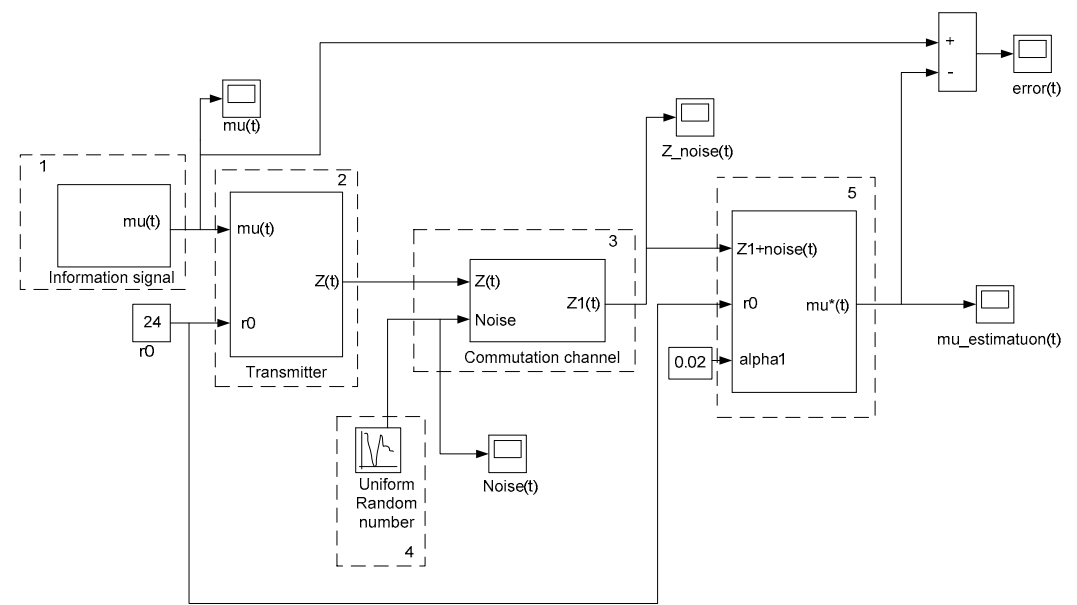

Figure 12. MATLAB/Simulink overall secure communication system block diagram with a two-channel synergetic observer. Here: block 1 is the useful signals source; block 2 is the transmitter with a chaotic generator; block 4 simulates errors in the communication channel; block 3 is the communication channel; and block 5 is the receiver (two-channel observer).

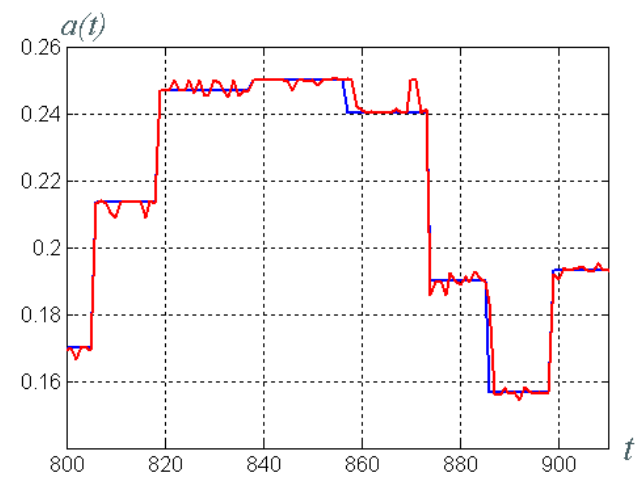

(a)

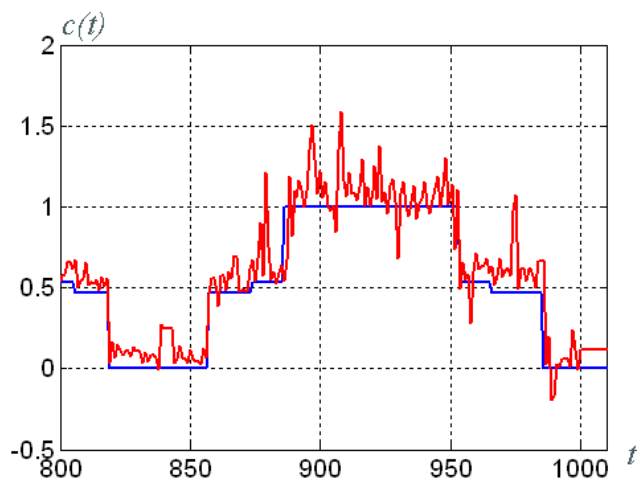

(b)

Figure 13. Useful signal reconstruction with a two channel observer: (a) useful signal $a$ (blue is the initial signal at the transmitter side; red is the reconstructed signal at the receiver side); (b) useful signal $c$ (blue is the initial signal at the transmitter side; red is the reconstructed signal at the receiver side).

In Figures 13 and 14, the simulation results of transmission and reconstruction of two piecewise-constant test signals are presented. In Figure 13a,b, the initial useful signals a and c are displayed as the blue lines, and the recovered signals are displayed via the red lines. We can see that the reconstructed signals had a form like the original ones but with an error. In order to illustrate the reconstruction error values, we displayed separate plots for reconstruction error values in Figure 14a,b. To compare the initial information signal with the transmitted one, we plotted the transmitted signal $\mathrm{Z}(\mathrm{t})$ with rectangular pulses in the communication channel encoded by the chaotic system in Figure 6 .

To demonstrate the operability of the proposed algorithm, we perform additional simulation of the resulting closed-loop system with an observer in another computer mathematics package, Maple. Here we introduce a sinusoidal signal to the input of the system and provide simulations on a different time interval.

Let us compare the simulation results in Figures 13 and 14 and Figures 15 and 16 . When simulating the reconstruction of a sinusoidal signal, it can be seen that along with the cases of Figures 13 and 14, a smaller error was achieved for channel $a$ 's signal transmission. In addition, at the initial time, the synergetic observer gave the largest recovery error. 


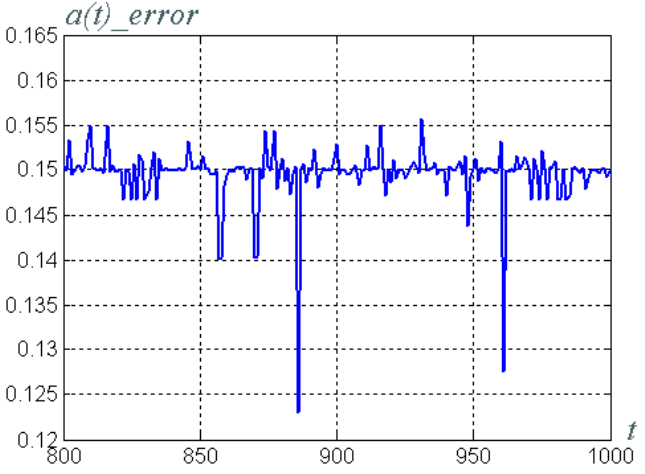

(a)

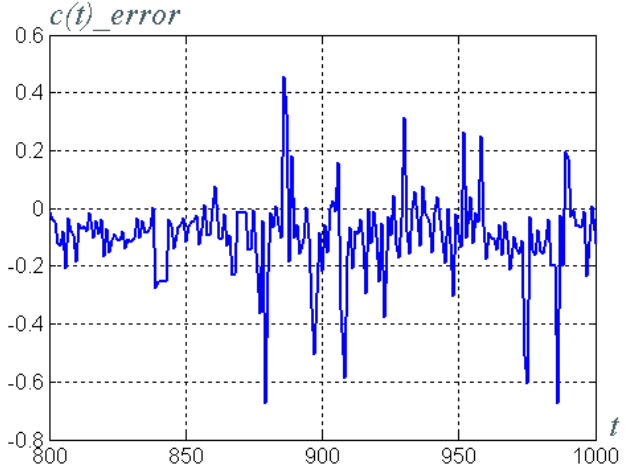

(b)

Figure 14. Transmitted signal reconstruction error: (a) Signal $a$ reconstruction error; and (b) Signal $c$ reconstruction error.

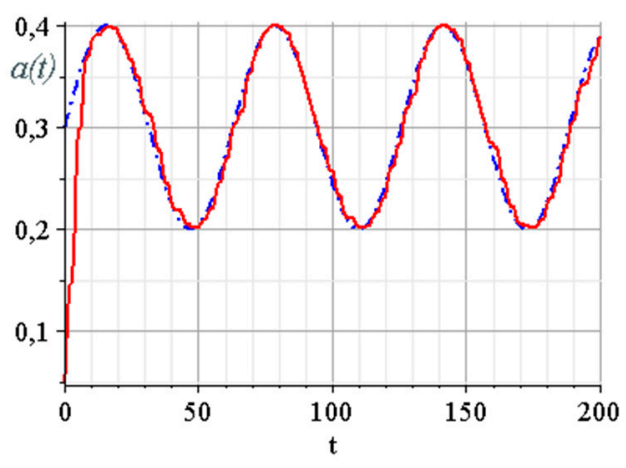

(a)

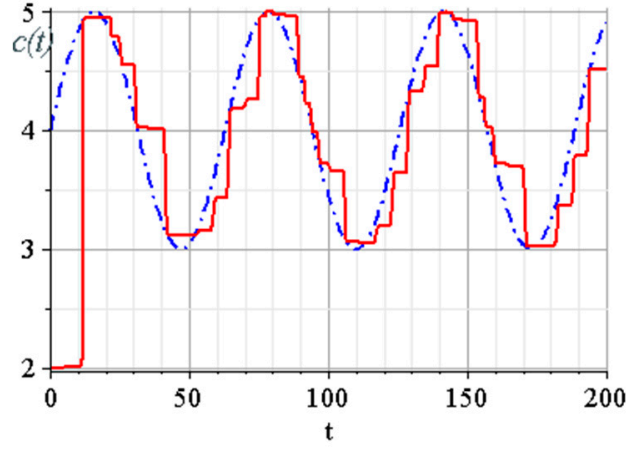

(b)

Figure 15. Sinusoidal signal reconstruction with a two channel observer: (a) Useful signal $a$ (blue is the initial signal at the transmitter side; red is the reconstructed signal at the receiver side); and (b) Useful signal $c$ (blue is the initial signal at the transmitter side; red is the reconstructed signal at the receiver side).

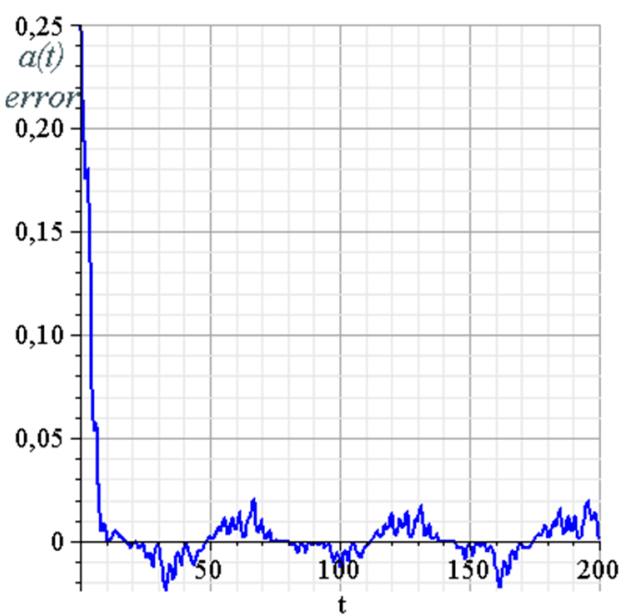

(a)

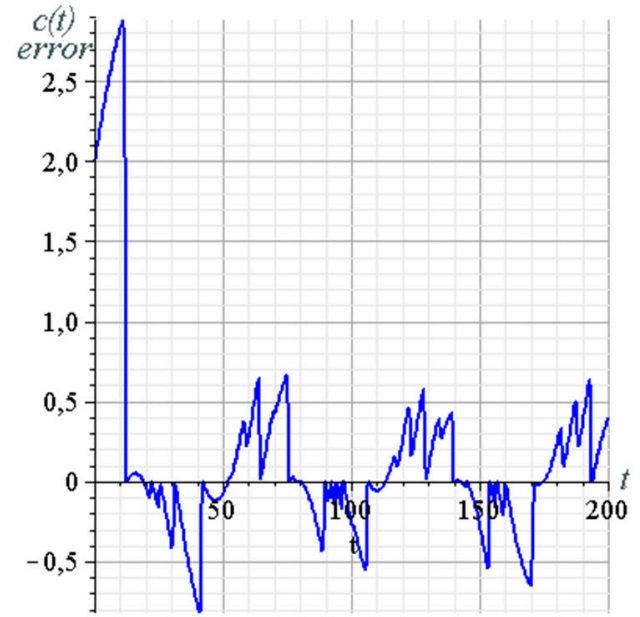

(b)

Figure 16. Sinusoidal signal reconstruction error: (a) Signal $a$ reconstruction error; and (b) Signal $c$ reconstruction error. 
The overall block diagram illustrating the approach to communication system design is shown in Figure 17.

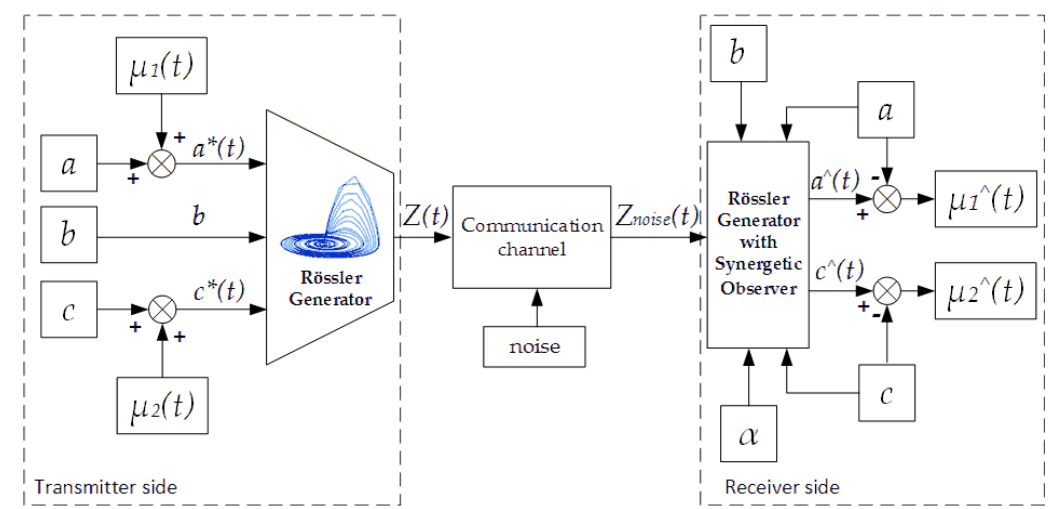

Figure 17. General block diagram of a synergetic observer communication system. Here: $a, b, c$ are the known parameters of the chaotic generator model; $a^{*}(t)$ and $c^{*}(t)$ are the modulated parameters; $Z(t)$ is the Rössler transformed system (2) variable; $Z_{\text {noise }}(t)$ is the $Z(t)$ variable with noises admixed in the transmission media; $a^{\wedge}(t)$ and $c^{\wedge}(t)$ are the estimated modulated parameters; $\mu_{1}(t)$ and $\mu_{2}(t)$ are the initial useful signals; $\mu_{1}{ }^{\wedge}(t)$ and $\mu_{2}{ }^{\wedge}(t)$ are the reconstructed useful signals; and $\alpha$ is the observer tuning parameter.

An algorithm for our synergetic observer design procedure is presented in Table 2. According to the above example of chaotic carrier communication system design and two-channel synergetic observer design procedure for a Rössler chaotic generator, we provide this algorithm in detail to provide its possible implementation for systems with other chaotic generators.

Table 2. Example of steps for our synergetic observer design procedure for Rössler generator.

\begin{tabular}{|c|c|}
\hline Design Step & Description \\
\hline Step 1 & $\begin{array}{l}\text { Deduce the system into a form suitable for transmitting a signal over a communication channel: for the initial } \\
\text { nonlinear system of the 3rd order, using the global reconstruction of a dynamic system [13] replace the } \\
\text { variables for transmitting a useful signal to the communication channel (Equation (3)). }\end{array}$ \\
\hline Step 2 & $\begin{array}{l}\text { Perform multiplicative parametric modulation: select two constant coefficients in different equations of the } \\
\text { original model and replace them with the sum of this coefficient and the time function that reflects the useful } \\
\text { signal (for each of the coefficients) (System (5)). }\end{array}$ \\
\hline Step 3 & $\begin{array}{l}\text { Adopt these sums as new unknown parameters and and extend the generator's initial model by dynamic } \\
\text { models of these parameters } \dot{w}_{1}(t)=0, \dot{w}_{2}(t)=0 \text {. As a result we get a } 5 \text { th order system with } 3 \text { observable }(x, y \\
\text { and } z) \text { and } 2 \text { unobservable }\left(w_{1} \text { and } w_{2}\right) \text { variables (Equation (9)). }\end{array}$ \\
\hline Step 4 & Introduce variables $\hat{w}_{1}$ and $\hat{w}_{2}$, which will be estimates of the parameters $w_{1}$ and $w_{2}$. \\
\hline Step 5 & $\begin{array}{l}\text { To find them we define the vector of the macro variable and write down the reduction equations with new } \\
\text { unknown functions that will be defined on the final steps of the procedure (Matrix (10)). }\end{array}$ \\
\hline Step 6 & Differentiating reduction equations with respect to time (System (12)). \\
\hline Step 7 & $\begin{array}{l}\text { Write down a main functional equation of the ADAR method }[38,40] \text { that will define the final system desired } \\
\text { dynamics. The } L \text { coefficients matrix will provide a desired system stability condition (Equation (13)). }\end{array}$ \\
\hline Step 8 & $\begin{array}{l}\text { From the main functional equation with respect to the extended initial mathematical model, the vector of the } \\
\text { macro variable, the reduction equations and its derivative we obtain the draft equations of the observer } \\
\text { (Equation (14)). }\end{array}$ \\
\hline Step 9 & $\begin{array}{l}\text { The observer equations should not contain unobservable state variables, therefore, from the draft observer } \\
\text { equations it is necessary to write out all terms containing unobservable variables and equate them to zero. We } \\
\text { thus get a system of the } 4 \text { th order (Equation (15)). }\end{array}$ \\
\hline Step 10 & $\begin{array}{l}\text { For the resulting system, we choose values of the coefficients } L \text { based on the stability condition of the observer } \\
\text { and the main functional equation (Equations }(16)-(18) \text { ). }\end{array}$ \\
\hline Step 11 & We obtain the observer Equation (19) taking into account the found functions for the reduction Equation (11). \\
\hline
\end{tabular}

\subsection{Alternative Reconstruction Method Demonstration}

To demonstrate the advantages of the synergetic nonlinear state observer design procedure in the subsections above, we considered an alternative method for reconstructing a useful signal on the receiver side. The recovery methods described in the previous sections and given in Table 1 did 
not provide applicability to nonlinear mathematical models of chaotic generators and simultaneous interdependent recovery of two parameters. The procedure for reconstructing a signal using an estimation of the least squares method (LS method) is described here.

Let us identify the parameters $a$ and $c$ with the recursive least squares method. For the system:

$$
y=B x
$$

the formula for determining unknown parameters in discrete form is the following matrix expression:

$$
B_{N+1}=B_{N}+\frac{P_{N}^{-1} x}{1+x^{T} P_{N}^{-1} x}\left(y_{N+1}-x^{T} B_{N}\right)
$$

where $\boldsymbol{B}$ is the vector of parameters to be defined; $\boldsymbol{x}$ is the vector of input variables; $y$ is the vector of output variables.

Next, we move from the discrete form of Equation (21) to the continuous one:

$$
\dot{B}(t)=\frac{R x}{\left(1+x^{T} R x\right)}\left(y-x^{T} B\right) \frac{1}{\Delta}
$$

where $\boldsymbol{R}=\boldsymbol{P}^{-1}, \boldsymbol{P}$ is the arbitrary matrix, $\Delta$ is the step of the system solution.

To build the $a$ and $c$ parameter identifiers of the mathematical model (1), we consider each of the equations separately. To design a parameter for observer $a$, we extract from model (1) an equation containing the following parameter:

$$
\frac{d y}{d t}=x+a y
$$

In this case, the input and output vectors will look like

$$
x=[y] ; y=[\dot{\hat{y}}(t)-x],
$$

where $\dot{\hat{y}}(t)$ is the evaluation of the derived variable $y$, which can be obtained by a control loop with two integrators:

$$
\begin{aligned}
& \dot{\mu}_{1}(t)=\mu_{2}+k_{1}\left(y-\mu_{1}\right), \\
& \dot{\mu}_{2}(t)=k_{2}\left(y-\mu_{1}\right)
\end{aligned}
$$

where $\mu_{2}=\dot{\hat{y}}(t)$ is the evaluation of the derived variable $y ; k_{1}$, and $k_{2}$ are the constant coefficients.

The vector of unknown coefficients is formed from estimates of the corresponding parameters, in our case:

$$
\boldsymbol{B}=[a] .
$$

As the initial values of the matrix $R$, we can take any numerical nonsingular matrix, but for better convergence of the parameter estimates we choose the following one:

$$
\boldsymbol{R}=\boldsymbol{X}^{T} \boldsymbol{X}=\left[R_{1}\right]
$$

where $\boldsymbol{X}=\left[\begin{array}{l}y\left(t_{k}\right) \\ y\left(t_{k+1}\right) \\ y\left(t_{k+2}\right)\end{array}\right]$ is the matrix formed by $t_{k}, t_{k+1}$ and $t_{k+2}$ countdowns.

Thus, from Equation (22) while taking into account Equations (23), (25), (26), we have:

$$
\dot{\hat{a}}=\frac{R_{1} y}{1+R_{1} y^{2}}(\dot{\hat{y}}-x-\hat{a} y) \frac{1}{\Delta} .
$$


In the same way for the parameter $c$ we get the following estimation:

$$
\dot{\hat{c}}=\frac{-R_{2} z}{1+R_{2} z^{2}}(\dot{\hat{z}}-b-x z+\hat{c} z) \frac{1}{\Delta} .
$$

The simulation plots of system of Equation (1) alongside with Equations (24), (27), (28) and the reconstructed information signal are shown in Figures 18 and 19.

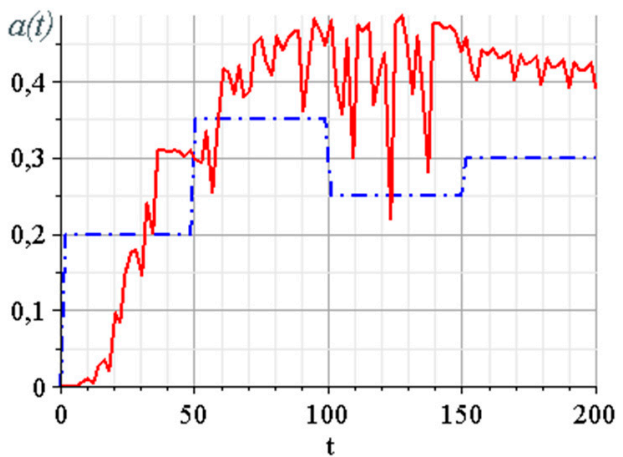

(a)

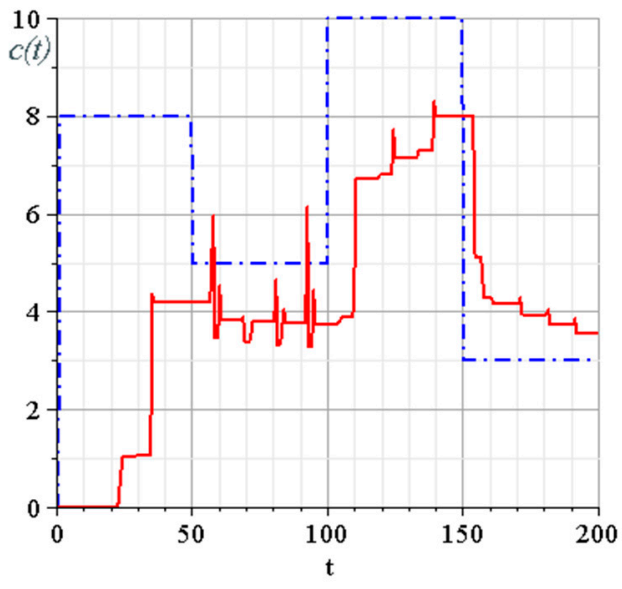

(b)

Figure 18. Useful signal reconstruction with a two channel LS method observer: (a) Useful signal $a$ (blue is the initial signal at the transmitter side; and red is the reconstructed signal at the receiver side); (b) Useful signal c (blue is the initial signal at the transmitter side; and red is the reconstructed signal at the receiver side).

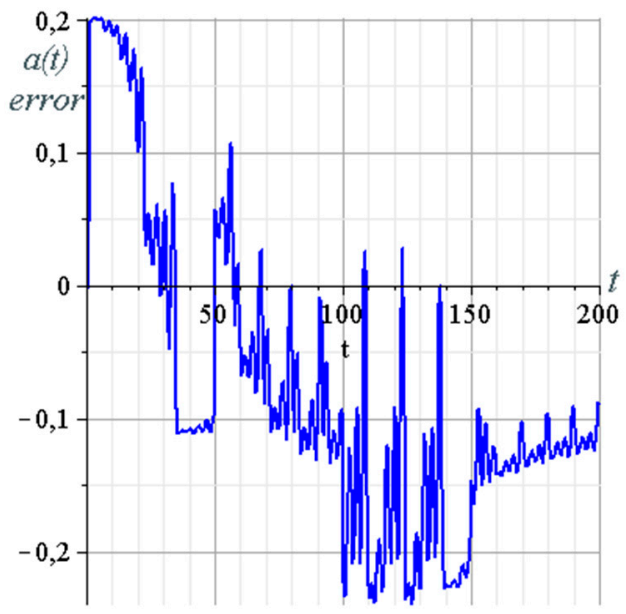

(a)

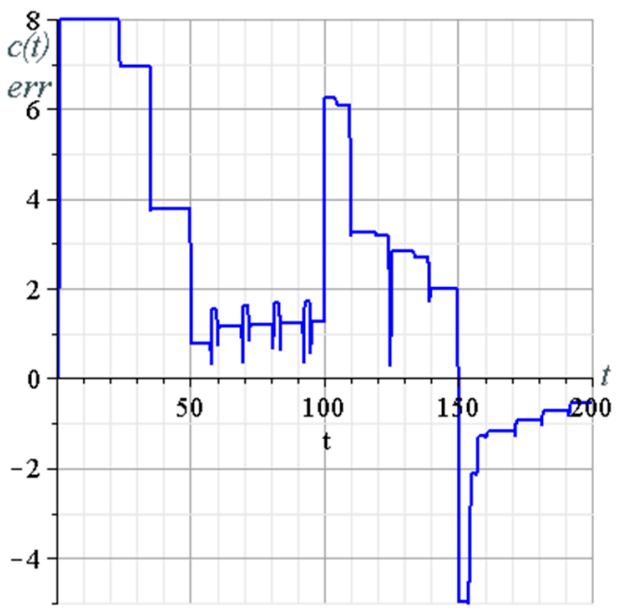

(b)

Figure 19. Transmitted signal LS method reconstruction error: (a) signal $a$ reconstruction error; and (b) signal $c$ reconstruction error.

The simulation results show a low efficiency of this estimation method for reconstructing a signal with a chaotic carrier. In particular, in Figures 18 and 19, an unacceptably high recovery error was observed for parameter $a$. In general, based on the results of a qualitative comparison of Figure 13, Figure 15 with Figure 18, we can outline the advantages of a synergetic nonlinear observer. In addition, in further modeling of the system of Equation (1) and Equations (24), (27), (28), the least squares method was inoperative due to the accumulation of estimation errors. 


\section{The System Hardware and Software Implementation Options}

In this study, the implementation of our new algorithms was performed by simulation in the MATLAB/Simulink and/or Maple environment. However, the obtained simulation results may have depended on MATLAB and/or Maple computation engine features. To test and validate the system, it is advisable to perform simulations on various software and/or hardware platforms.

\subsection{Brief Overview of System Hardware Implementation Issues}

The chaotic systems, as well as chaotic communication systems, may be hardware-implemented by using different kinds of embedded systems, for example: Arduino, Raspberry Pi and FPGAs.

Digital implementation of the four-dimension autonomous hyperchaotic system on a Field Programmable Gate Array (FPGA) for creating a chaotic secure communication system using a XILINX VERTEX5 chip recently reported by Tami et al. [14]. Bit error rate analysis in FPGA/FPAA based communication systems by using Arduino in a Matlab/Simulink environment with Sprott chaotic generator performed by Günay and Altun in [15]. The approach of chaotic system simulation in the Arduino platform is described by Silva, et al. in [16]. Dual-channel Chaotic Generator of Mackey-Glass system is implemented on Arduino Due in [17] by Voliansky et al. A Raspberry Pi 3 implementation of Lorenz Chaotic System with estimation by Hybrid Jaya-Powell Algorithm is described by Zhuang, et al. in [18]. This small overview defines several validated options of chaotic system hardware implementation, while a synergetic observer is a resource-intensive computational algorithm and requires additional research to find a suitable platform. The most promising and universal approach is to program a designed synergetic observer in FPGAs. For simulation, the resulting closed-loop system can be represented in a form suitable for numerical simulation. FPGA implementation techniques for chaotic systems are described by Tlelo-Cuautlea, et al. [20], by Guillen-Fernandezb et al. [19] and in other related papers. Notably, Tlelo-Cuautlea, et al. [20] demonstrate the implementation of Forward Euler and Runge-Kutta numerical methods on an FPGA. Guillen-Fernandezb et al. [19] demonstrate synchronization techniques examples applied to 22 chaotic oscillators. These implementation examples may be used in a hardware implementation of the designed system from Sections 3.5 and 3.6.

\subsection{Example of Software Implementation on a Standard PC without MATLAB Computational Engine}

The promising one is a model-oriented approach to modeling when the system created in MATLAB/Simulink is transformed into code in a high-level language (for example, $\mathrm{C}++$ ), and then it is executed as a separate application program on a standard PC or compatible embedded system. We used this approach when modeling a single-channel data transmission system with a chaotic carrier created in our earlier work [43].

In order to validate the system for transmission of useful digital data, our team with the help of Alexander D. Zolkin converted the MATLAB/Simulink system diagrams into C++ code and used it to create the virtual test bed providing transmission of a test phrase. The main window of the testbed is presented at Figure 20a,b. The test phrase is "Lorem ipsum dolor sit amet, consectetur adipiscing elit". As expected, the error of signal reconstruction in the proposed algorithm did not allow it to be used to transfer useful digital data without additional processing. As we needed only to validate the data transmission system, we only used multiple transmission as the simplest method of eliminating transmission errors. Figure 20a shows the result of the transmission with a number of retransmissions of 20,000.

A smaller number of transmissions, for example, 8000, resulted in the persistence of transmission errors (Figure 20b). We also evaluated the necessary number of repetitions to provide the desired error rate in transmission. However, this issue requires future study. The same procedure may be used for two-channel communication system validation. 


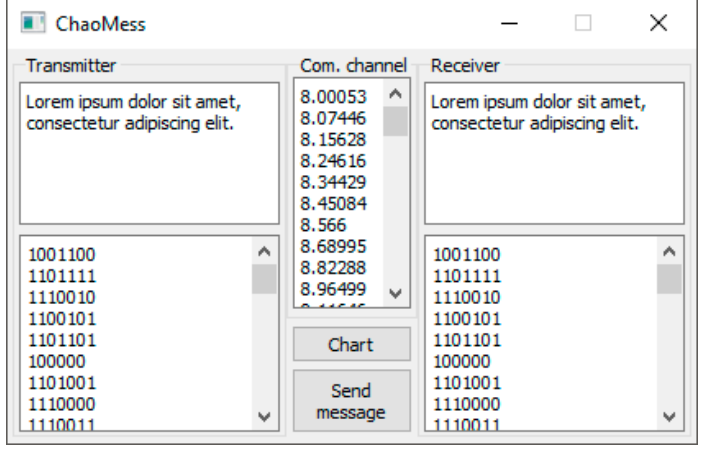

(a)

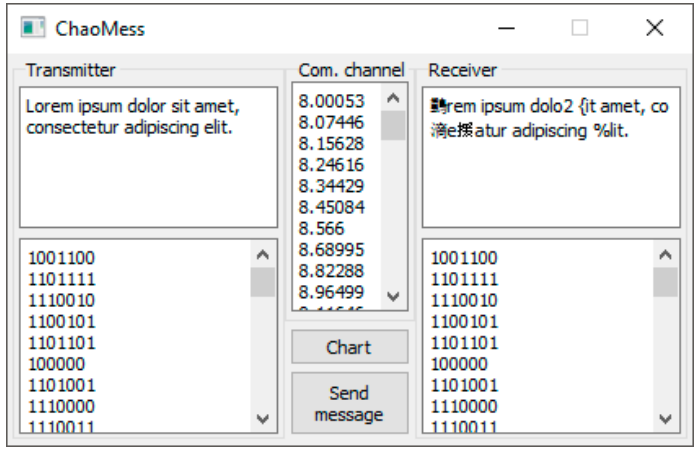

(b)

Figure 20. Testbed main window after message delivery. (a) number of iterations: 20,000; (b) number of iterations: 8000 .

The simulation software system used the simplest error correction algorithm: multiple transmission. This algorithm was chosen only to demonstrate the feasibility of the system with a synergetic observer to practical implementation for transmitting binary data. In Figure 21, we show the results of a statistical analysis of the created software system, having studied the dependence of the number of repetitions on the number of errors. The dependencies in Figure 21 make it possible to identify the best combination of the number of retries and the transmission rate that was used in the simulation results in Figure 20.

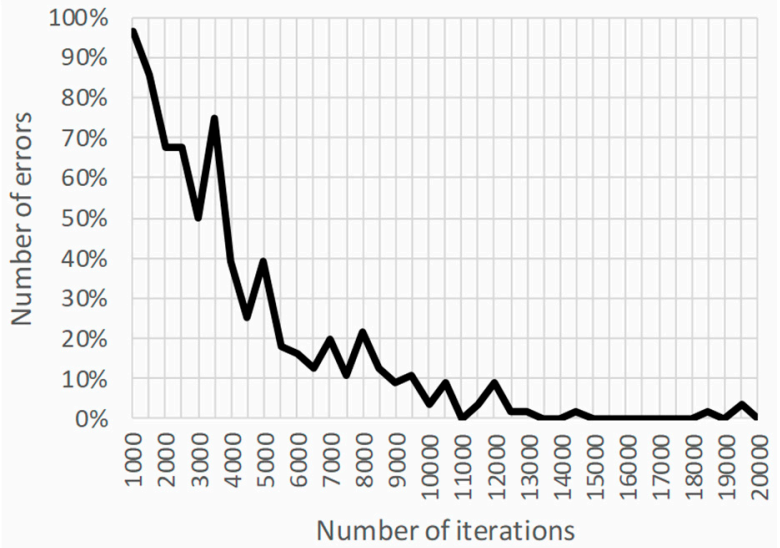

(a)

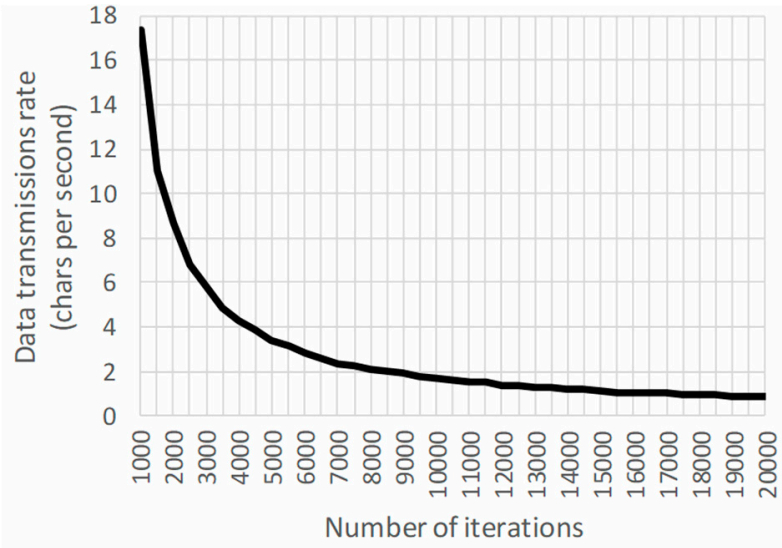

(b)

Figure 21. Statistical analysis after message delivery. (a) Error rate vs. number of transmission repetitions; and (b) Transmission speed vs. number of transmission repetitions.

\subsection{Example of Black-and-White Image Transmission with MATLAB Computational Engine}

The encryption system described in the previous subsection (Figures 12 and 17) can be used to encode any type of information, including graphic information, which is one of the most popular application examples for chaotic data transmission. Therefore, to illustrate the operability of this system, we considered confidential image transmission. As a test image, a black-and-white image $200 \times 200$ pixels in size with 256 shades of gray level (a portrait of the writer Anton P. Chekhov (1860-1904)), shown in Figure 22a, was selected.

When transmitting images, the information signal is a two-dimensional function $f(x, y)$, where $x$ and $y$ are the spatial coordinates, and the amplitude $f$ is the intensity (brightness) or gray level at any point with a pair of coordinates $(x, y)$. 


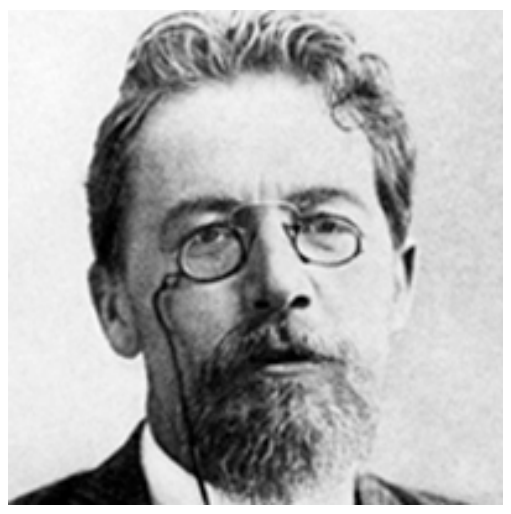

(a)

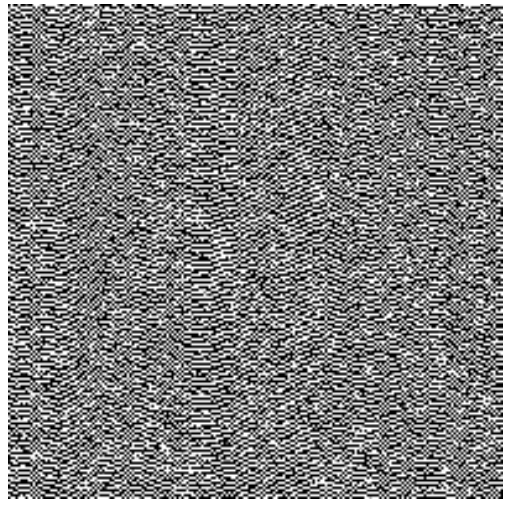

(b)

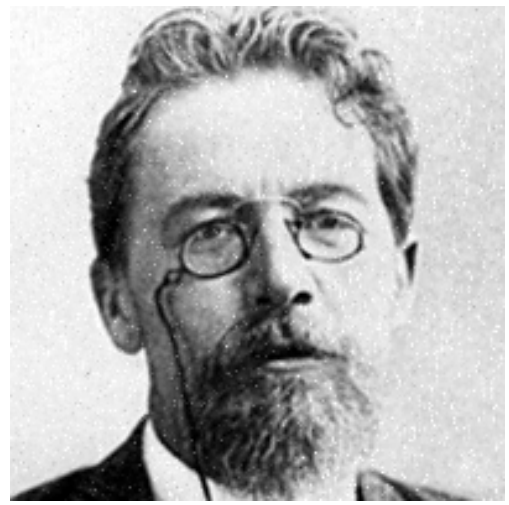

(c)

Figure 22. Test images: (a) Test black and white image with 256 gradations of the gray level at the system input; (b) Signal in the communication channel; (c) Restored image.

To submit an image to the system input, we used a block From file to receive data from an external file. The data are presented in the form of a matrix

$$
\left[\begin{array}{cccc}
t_{1} & t_{2} & \ldots & t_{\text {end }} \\
u_{1} & u_{2} & \ldots & u_{\text {end }}
\end{array}\right]
$$

where the first row of the matrix represents time samples in ascending order, and the second one is the image elements (pixels) at these points in time.

To convert a BMP image into data of the form in Equation (1), it is necessary to execute a script in the workspace of the MATLAB system as a standard MATLAB procedure from the digital image processing libraries. The input of the script is the initial image BMP-file, the output is a MATLAB data MAT-file. After simulation of the image's transmission, the script is executed in reverse order to generate an output BMP-image file.

Model parameters as per Equation (1) can be changed in the model topology file, or through the graphical user interface in the MATLAB/Simulink system. During the simulation, the following parameters were selected:

- fixed parameters of the Rössler system: $a=b=0.2$;

- the parameter of the synergetic observer that determines the rate of estimation of the parameter,

$$
\alpha=0.01 \text {; and }
$$

- the modulating signal is presented in the form of a stepwise time dependence, the height of each step corresponding to the gray level of the black-and-white image.

The resulting restored image is shown in Figure 22c and the encoded signal in the communication channel shown in Figure 22b.

\section{Discussion}

The designed system is presented in the form of a MATLAB/Simulink model, and the results of computer modeling of the constructed system are presented above. The simulation results confirm the viability of our approach. Moreover, earlier we have studied the cryptographic strength and steganography for another 3rd order chaotic system with parametric modulation of only one parameter introduced into the system in a non-linear manner, i.e., multiplicatively using the autocorrelation method and the Fourier transform. As a result, autocorrelation has shown the complete identity of the signal analysis results with and without the added useful signal component. Analysis by the Fourier transform method has shown that the difference in the level of the leading frequency of the Fourier transform spectrograms is only $0.002 \mathrm{~Hz}$ for signals with and without the useful information 
component, which will not allow detecting the existence of the useful signal by an external listener. In our current studies, we have applied these methods, validated in previous research to build the two-channel transmission system presented here.

The approach to low-level protection of transmitted data used in this work is extremely effective and, with the exception of cases of the simplest implementation of a coding and communication system, allows good protection of the transmitted information [12,13], including hiding the data and even the fact of its transmission, not allowing a malicious observer to determine or extract any useful signal from the noise in the communication channel. This idea was repeatedly considered in the work of various previous authors, however the implementation of this approach turned out to be difficult due to a number of problems with decryption of such a protected signal on the receiver side. A general description of the use of synergetic observers in systems with chaotic dynamics is given by Kolesnikov et al. [39], and the procedure for a single-channel system was presented in previous research [42,43]. In this regard, the present study, which allows developing a two-channel reconstruction technique for two useful signals masked by chaotic oscillations on the receiving side, can provide a practical implementation of a very promising and practical approach for developing new communication systems for transmitting signals with a chaotic carrier.

In this study we did not use the simplest forms of parametric modulation. In many other well-known works using the parametric modulation method the useful signal is added to the model of the chaotic generator as an additive component (e.g., [46]), but we use an approach in which the useful signal is added as a multiplicative component, i.e., we use "non-linear mixing", which will have greater security. Our procedure applied to the 3rd order model of the chaotic system. According to Figure 13, we can see a reconstruction error rate up $25 \%$ of the original signal or even more, but mainly this error has the form of a short-time disturbance and may be eliminated with basic smoothing and filtration algorithms.

\section{Conclusions}

The paper presented a design procedure for a two-channel synergetic state observer for a system of secured information transmission with a chaotic carrier. A system based on a synergetic state observer has the following advantages: (i) it allows estimation of up to two signals simultaneously; (ii) it does not require chaotic synchronization of the receiver and transmitter; (iii) it does not require determining the Lyapunov function; (iv) the observer structure is not rigid, the structure is not postulated in advance, but is determined during the design procedure based on the model of a chaotic generator; and (v) it provides direct application to systems with nonlinear dynamics without linearization of generator's nonlinear model.

The simulation results confirmed the viability of the proposed procedure and the capability of organizing a system of chaotic information transmission. A qualitative assessment of the image transmission results according to Figure 13 confirms the possibility of secure image transmission with sufficient reliability because the quantitative value of the error plays a secondary role in such tasks. During the test transmission of rectangular pulses (the simulation results in Figure 13a,b a significant signal recovery error is observed; the recovery error can reach $25 \%$ of the amplitude of the useful signal. However, the error is pulsed, so its presence does not interfere with the conclusion about the original waveform. Moreover, such an error can be eliminated by additional signal processing using digital filtering and smoothing algorithms.

It seems that additional research on the accuracy of the useful signal recovery is needed. According to Figures 13 and 22, the receiver quality may be acceptable for the transmission of some images, but in the case of numerical message transmission, a procedure of error detection and a recovery process is necessary. One potential solution is to make the frequency of the useful signal significantly lower than the fundamental frequency of oscillations of the chaotic system. In addition, future research should make an extra study to explore the impact of the observer's tune parameter $\alpha$ not only for speed of estimation but for receiver quality. It is reasonable to explore application of some smoothing and 
filtration algorithms in order to decrease or eliminate a useful signal reconstruction error at the receiver side on the output of the synergetic observer. Meanwhile, the receiver error may be also related to the MATLAB system solver. It thus may be necessary to repeat the simulation in LabView or in a future hardware-implemented system. In addition, errors can occur due to external influences on the communication channel. Thus, one way to increase the accuracy is by including digital filtration algorithms into the system schematic.

In further studies, it will be useful to numerically compare the resulting synergetic observer with other methods of signal recovery. In addition, as we introduce the useful signal as a function directly into the structure of the generator of chaotic oscillations multiplicatively, another topic for further research may be a deep estimation of actual steganography of the transmitted signal on the output of the chaotic generator.

Author Contributions: Conceptualization, A.M.; methodology, A.M.; software, J.D.; validation, A.M., J.D., A.N., and C.F.; formal analysis, A.M.; investigation, A.M., J.D., A.N., and C.F.; resources, A.M.; data curation, J.D.; writing - original draft preparation, A.M. and J.D.; writing—review and editing, A.M., A.N. and C.F.; visualization, J.D.; supervision, A.N. and C.F.; project administration, A.M.; funding acquisition, A.M. All authors have read and agreed to the published version of the manuscript.

Funding: This work was funded by the Southern Federal University.

Conflicts of Interest: The authors declare no conflicts of interest. The funders had no role in the design of the study; in the collection, analyses, or interpretation of data; in the writing of the manuscript, and in the decision to publish the results.

\section{References}

1. Lorenz, E.N. Deterministic nonperiodic flow. J. Atmos. Sci. 1963, 20, 130-141. [CrossRef]

2. Lorenz, E.N. Can chaos and intransitivity lead to interannual variability? Tellus A Dyn. Meteorol. Oceanogr. 1990, 42, 378-389. [CrossRef]

3. Boccaletti, S.; Kurths, J.; Osipov, G.V.; Valladares, D.; Zhou, C. The synchronization of chaotic systems. Phys. Rep. 2002, 366, 1-101. [CrossRef]

4. Geneser, S.; Kirby, R.; Sachse, F.B. Sensitivity Analysis of Cardiac Electrophysiological Models Using Polynomial Chaos. IEEE Eng. Med. Biol. 2005, 4, 4042-4045.

5. Wang, L.; Feng, G. Analysis of a Predator-Prey System Concerning Impulsive Effect. Int. Workshop Chaos-Fractals Theor. Appl. 2009, 93-97.

6. Cui, H.; Song, X. Research on Electricity Price Forecasting Based on Chaos Theory. Int. Semin. Future Inf. Technol. Manag. Eng. 2008, 398-401.

7. Kiel, L.D.; Elliott, E. Chaos Theory in the Social Sciences: Foundations and Applications; University of Michigan Press: Ann Arbor, MI, USA, 1996; p. 360. [CrossRef]

8. Nakamura, Y.; Sekiguchi, A. The chaotic mobile robot. IEEE Trans. Robot. Autom. 2001, 17, 898-904. [CrossRef]

9. Feki, M. An adaptive chaos synchronization scheme applied to secure communication. Chaos, Solitons Fractals 2003, 18, 141-148. [CrossRef]

10. Murali, K.; Lakshmanan, M. Secure communication using a compound signal from generalized synchronizable chaotic systems. Phys. Lett. A 1998, 241, 303-310. [CrossRef]

11. Zaher, A.; Abu-Rezq, A. On the design of chaos-based secure communication systems. Commun. Nonlinear Sci. Numer. Simul. 2011, 16, 3721-3737. [CrossRef]

12. Dmitriev, A.S.; Panas, A.I. Dynamic Chaos. New Data Media for Communication Systems; PHISMATLIT: Moscow, Russia, 2002; 251p. (In Russian)

13. Anishchenko, V.S.; Pavlov, A.N.; Yanson, N.B. Reconstruction of dynamic systems as applied to secure communications. Tech. Phys. 1998, 43, 1401-1407. [CrossRef]

14. Tami, T.; Messaoudene, T.; Ferdjouni, A.; Benzineb, O. Chaos secure communication' implementation in FPGA. In Proceedings of the 2018 International Conference on Applied Smart Systems (ICASS), Medea, Algeria, 24-25 November 2018; pp. 1-6. 
15. Gunay, E.; Altun, K. BER analysis and application in FPGA and FPAA based communication systems. In Proceedings of the 2017 International Artificial Intelligence and Data Processing Symposium (IDAP), Malatya, Turkey, 16-17 September 2017; pp. 1-5.

16. Silva, P.H.O.; Nepomuceno, E.G.; Vitorino, A.; Martins, S.A.M. A visual chaotic system simulation in Arduino platform controlled by Android app. In Proceedings of the 2017 IEEE World Engineering Education Conference (EDUNINE), Santos, Brazil, 19-22 March 2017; pp. 62-66.

17. Voliansky, R.; Sadovoi, O.; Shramko, Y.; Volianska, N.; Sinkevych, O. Arduino-based Implementation of the Dual-channel Chaotic Generator. In Proceedings of the 2019 3rd International Conference on Advanced Information and Communications Technologies (AICT), Lviv, Ukraine, 2-6 July 2019; pp. 278-281.

18. Zhuang, L.; Cao, L.; Wu, Y.; Zhong, Y.; Zhangzhong, L.; Zheng, W.; Wang, L. Parameter Estimation of Lorenz Chaotic System Based on a Hybrid Jaya-Powell Algorithm. IEEE Access 2020, 8, 20514-20522. [CrossRef]

19. Guillén-Fernández, O.; Meléndez-Cano, A.; Tlelo-Cuautle, E.; Núñez-Pérez, J.C.; Rangel-Magdaleno, J. On the synchronization techniques of chaotic oscillators and their FPGA-based implementation for secure image transmission. PLoS ONE 2019, 14, e0209618. [CrossRef] [PubMed]

20. Tlelo-Cuautle, E.; Rangel-Magdaleno, J.; Pano-Azucena, A.; Obeso-Rodelo, P.; Nuñez-Perez, J.-C. FPGA realization of multi-scroll chaotic oscillators. Commun. Nonlinear Sci. Numer. Simul. 2015, 27, 66-80. [CrossRef]

21. Riaz, A.; Ali, M. Chaotic Communications, their applications and advantages over traditional methods of communication. In Proceedings of the 2008 6th International Symposium on Communication Systems, Networks and Digital Signal Processing, Graz, Austria, 25 July 2008; pp. 21-24.

22. Short, K.M. Unmasking a modulated chaotic communications scheme. Int. J. Bifurc. Chaos 1996, 6, 367-375. [CrossRef]

23. Yang, H.; Tang, W.K.; Chen, G.; Jiang, G.-P. System Design and Performance Analysis of Orthogonal Multi-Level Differential Chaos Shift Keying Modulation Scheme. IEEE Trans. Circuits Syst. I Regul. Pap. 2016, 63, 146-156. [CrossRef]

24. Cheng, G.; Xu, W.; Chen, C.; Wang, L. SWIPT Schemes for Carrier Index Differential Chaos Shift Keying Modulation: A New Look at the Inactive Carriers. IEEE Trans. Veh. Technol. 2019, 68, 2557-2570. [CrossRef]

25. Wang, S.F. Dynamical Analysis of Memristive Unified Chaotic System and Its Application in Secure Communication. IEEE Access 2018, 6, 66055-66061. [CrossRef]

26. Isaeva, O.B.; Jalnine, A.Y.; Kuznetsov, S.P. Chaotic communication with robust hyperbolic transmitter and receiver. In Proceedings of the 2017 Progress in Electromagnetics Research Symposium-Spring (PIERS), St. Petersburg, Russia, 22-25 May 2017; pp. 3129-3136.

27. Andrievsky, B.; Fradkov, A.L. Adaptive-based methods for information transmission by means of chaotic signal source modulation. Autom. Remote. Control. 2011, 72, 1967-1980. [CrossRef]

28. Andrievsky, B. Information transmission based on adaptive synchronization of chaotic Lorenz systems over the digital communication channel. Cybern. Phys. 2013, 2, 10-14.

29. Fradkov, A.L.; Pogromsky, A.Y. Introduction to Control of Oscillations and Chaos; World Scientific Pub. Co.: Singapore, 1998; 391p.

30. Tyukin, I.Y.; Prokhorov, D.V.; Van Leeuwen, C. Adaptation and Parameter Estimation in Systems with Unstable Target Dynamics and Nonlinear Parametrization. IEEE Trans. Autom. Control. 2007, 52, 1543-1559. [CrossRef]

31. Chien, T.-H.; Chen, Y.-C. Combination of Observer/Kalman Filter Identification and Digital Redesign of Observer-Based Tracker for Stochastic Chaotic Systems. In Proceedings of the 2016 International Symposium on Computer, Consumer and Control (IS3C), Xi'an, China, 4-6 July 2016; pp. 103-107.

32. Wei, W.; Guo, L. Chaos control in AFM via disturbance observer based control. In Proceedings of the 2016 35th Chinese Control Conference (CCC), Chengdu, China, 27-29 July 2016; pp. 869-872.

33. Zhang, J.; Zhu, F. Chaos synchronization and chaos-based secure communication based on new unknown input observer approach. In Proceedings of the 2016 35th Chinese Control Conference (CCC), Chengdu, China, 27-29 July 2016; pp. 1891-1896.

34. Xiao, X.-S.; Pan, C. Observer-based adaptive synchronization of uncertain time-delay chaotic systems. In Proceedings of the 201736th Chinese Control Conference (CCC), Dalian, China, 26-28 July 2017; pp. 3321-3324. 
35. Datcu, O.; Stanciu, M.; Udrea, R.M. Secret speech transmission using a high order sliding mode observer. In Proceedings of the 2017 40th International Conference on Telecommunications and Signal Processing (TSP), Barcelona, Spain, 5-7 July 2017; pp. 161-164.

36. Moysis, L.; Volos, C.; Pham, V.-T.; Goudos, S.; Stouboulos, I.; Gupta, M.K. Synchronization of a Chaotic System with Line Equilibrium using a Descriptor Observer for Secure Communication. In Proceedings of the 2019 8th International Conference on Modern Circuits and Systems Technologies (MOCAST), Thessaloniki, Greece, 13-15 May 2019; pp. 1-4.

37. Gupta, M.; Tomar, N.K.; Mishra, V.K.; Bhaumik, S. Observer Design for Semilinear Descriptor Systems with Applications to Chaos-Based Secure Communication. Int. J. Appl. Comput. Math. 2017, 3, 1313-1324. [CrossRef]

38. Kolesnikov, A.A. Introduction of synergetic control. In Proceedings of the 2014 American Control Conference, Portland, OR, USA, 4-6 June 2014; pp. 3013-3016.

39. Kolesnikov, A.A.; Veselov, G.E.; Kolesnikova, S.I.; Kolesnikov, A.A.; Dzuba, J.N. Synergetic-cybernetic approach to creation of chaos-dynamics systems of data processing. Inf. Technol. Mag. 2013, 10, 1-32. (In Russian)

40. Kolesnikov, A.A. Synergatic Methods of Control of Complex Systems: Theory of System Synthesis, 3rd ed.; URSS: Moscow, Russia, 2019; 240p, ISBN 978-5-397-06702-7. (In Russian)

41. Kolesnikov, A.A. Analiticheskij sintez nelinejnyh sistem, optimal'nyh otnositel'no linejnyh agregirovannyh peremennyh. Elektromehanika 1985, 11, 71-79.

42. Mushenko, A.; Dzuba, J.N.; Zolkin, A.D. Recovering of useful signal in chaotic carrier data transmission system with state observer. In Proceedings of the 2018 Moscow Workshop on Electronic and Networking Technologies (MWENT), Moscow, Russia, 14-16 March 2018; pp. 1-5.

43. Kolesnikov, A.A.; Mushenko, A.; Dzuba, J.N.; Zolkin, A.D. Synergetic observer of state variables in problems of reconstruction of systems with chaotic dynamics. In Proceedings of the 2017 IEEE II International Conference on Control in Technical Systems (CTS), St. Petersburg, Russia, 25-27 October 2017; pp. 55-58.

44. Rossler, O. An equation for continuous chaos. Phys. Lett. A 1976, 57, 397-398. [CrossRef]

45. Flajolet, P.; Sedgewick, R. Analytic Combinatorics by Philippe Flajolet; Cambridge University Press (CUP): Cambridge, UK, 2009; p. 826, ISBN 978-0-521-89806-5.

46. Smaoui, N.; Karouma, A.; Zribi, M.A. Secure communications based on the synchronization of the hyperchaotic Chen and the unified chaotic systems. Commun. Nonlinear Sci. Numer. Simul. 2011, 16, 3279-3293. [CrossRef] 